\title{
Deficiency of macrophage PHACTR1 impairs efferocytosis and promotes atherosclerotic plaque necrosis
}

\author{
Canan Kasikara, ${ }^{1}$ Maaike Schilperoort, ${ }^{1}$ Brennan Gerlach, ${ }^{1}$ Chenyi Xue, ${ }^{1}$ Xiaobo Wang, ${ }^{1}$ Ze Zheng, ${ }^{2}$ George Kuriakose, ${ }^{1}$ \\ Bernhard Dorweiler, ${ }^{3}$ Hanrui Zhang, ${ }^{1}$ Gabrielle Fredman, ${ }^{4}$ Danish Saleheen, ${ }^{1}$ Muredach P. Reilly, ${ }^{1,5}$ and Ira Tabas ${ }^{1,6,7}$ \\ 'Department of Medicine, Columbia University Irving Medical Center, New York, New York, USA. ${ }^{2}$ Department of Medicine, Medical College of Wisconsin, Milwaukee, Wisconsin, USA. ${ }^{3}$ Department of Vascular \\ Surgery, University of Cologne, Cologne, Germany. ${ }^{4}$ Department of Molecular and Cellular Physiology, Albany Medical Center, Albany, New York, USA. Irving Institute for Clinical and Translational Research, \\ Columbia University Irving Medical Center, New York, New York, USA. ${ }^{6}$ Department of Physiology and Cellular Biophysics and 'Department of Pathology and Cell Biology, Columbia University Irving Medical \\ Center, New York, New York, USA
}

\begin{abstract}
Efferocytosis, the process through which apoptotic cells (ACs) are cleared through actin-mediated engulfment by macrophages, prevents secondary necrosis, suppresses inflammation, and promotes resolution. Impaired efferocytosis drives the formation of clinically dangerous necrotic atherosclerotic plaques, the underlying etiology of coronary artery disease (CAD). An intron of the gene encoding PHACTR1 contains rs9349379 (A>C), a common variant associated with CAD. As PHACTR1 is an actinbinding protein, we reasoned that if the rs9349379 risk allele $\mathrm{G}$ causes lower PHACTR1 expression in macrophages, it might link the risk allele to CAD via impaired efferocytosis. We show here that rs9349379-G/G was associated with lower levels of PHACTR1 and impaired efferocytosis in human monocyte-derived macrophages and human atherosclerotic lesional macrophages compared with rs9349379-A/A. Silencing PHACTR1 in human and mouse macrophages compromised AC engulfment, and Western diet-fed LdIr/- mice in which hematopoietic Phactr1 was genetically targeted showed impaired lesional efferocytosis, increased plaque necrosis, and thinner fibrous caps - all signs of vulnerable plaques in humans. Mechanistically, PHACTR1 prevented dephosphorylation of myosin light chain (MLC), which was necessary for AC engulfment. In summary, rs9349379-G lowered PHACTR1, which, by lowering phospho-MLC, compromised efferocytosis. Thus, rs9349379-C may contribute to CAD risk, at least in part, by impairing atherosclerotic lesional macrophage efferocytosis.
\end{abstract}

\section{Introduction}

Despite the availability of safe and effective risk-reducing therapies, coronary artery disease (CAD) remains the leading cause of morbidity and mortality in the United States and other developed countries (1). Ischemic CAD is caused by atherosclerosis, a lipoprotein-driven, nonresolving inflammatory process in which lipid, macrophages, other immune cells, and extracellular matrix accumulate in the arterial subendothelial space (intima) of large arteries at sites of disturbed blood flow (2-6). A distinguishing feature of clinically significant advanced atherosclerotic plaques is impaired macrophage clearance of dead cells (efferocytosis) (710). Impaired clearance of lesional apoptotic cells (ACs) by lesional macrophages through the process of efferocytosis promotes plaque necrosis, lesional inflammation, and defective inflammation resolution (7-10), all of which are associated with unstable atherosclerotic plaques and acute CAD (11-14). While observations in human advanced lesions and molecular-genetic causation studies in mice have supported this scenario, insight from human genetics in this area has been limited even though genome-wide association studies (GWAS) have identified many CAD-risk loci (15-21). Indeed,

Conflict of interest: The authors have declared that no conflict of interest exists. Copyright: (5) 2021, American Society for Clinical Investigation.

Submitted: November 20, 2020; Accepted: February 23, 2021; Published: April 15, 2021.

Reference information: J Clin Invest. 2021;131(8):e145275.

https://doi.org/10.1172/JCl145275. the application of human genetics to macrophage cell biological mechanisms in atherosclerosis remains an understudied area (18), and one of the challenges in this area is that most of the identified CAD-risk loci reside in noncoding regions and have uncertain links to the genes to which they are assigned (15-21). Concerning efferocytosis, Kojima et al. (22) showed that the 9p21 CAD-risk locus was associated with lower expression in smooth muscle cells of cyclin-dependent kinase inhibitor 2B (CDKN2B) and calreticulin. Calreticulin helps facilitate the recognition of ACs by macrophage LDL receptor-related protein 1 (LRP1), and the authors showed that the silencing of CDKN2B in smooth muscle cells led to decreased engulfment of apoptotic smooth muscle cells by macrophages in vitro. However, CAD-risk loci that directly affect efferocytosis processes in macrophages themselves have not yet been identified.

Several independent GWAS have shown that certain intronic variants in the gene encoding phosphatase and actin regulator 1 (PHACTR1) on chromosome 6p24 are associated with CAD risk (19, 23-26). Two independent fine-mapping studies have concluded that rs9349379 $(A>G)$, a common variant located in the third intron of PHACTR1, is the causal CAD-risk variant and that there are no secondary signals at this locus $(27,28)$. However, there is a discrepancy in whether PHACTR1 is the causal gene at this locus. One prior study has suggested that the G allele at rs9349379 increases CAD risk by causing increased expression of the gene encoding endothelin-1, EDN1, in endothelial cells without affecting PHACTR1 expression (28). However, separate work present- 
A

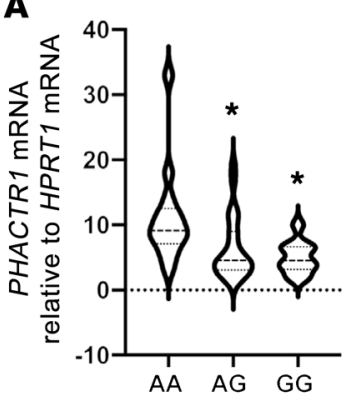

B

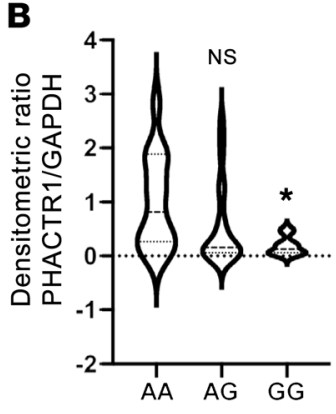

C

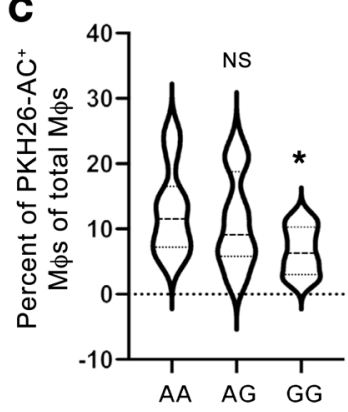

D

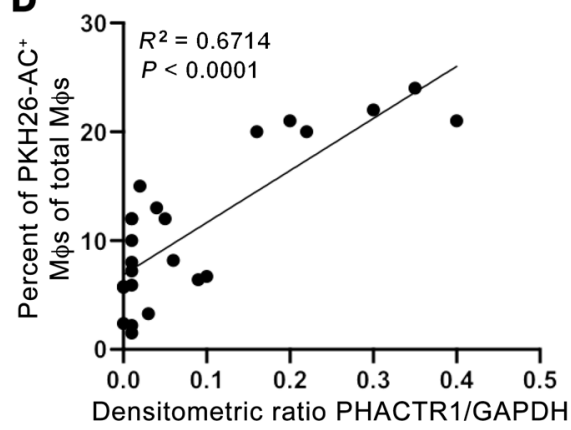

E
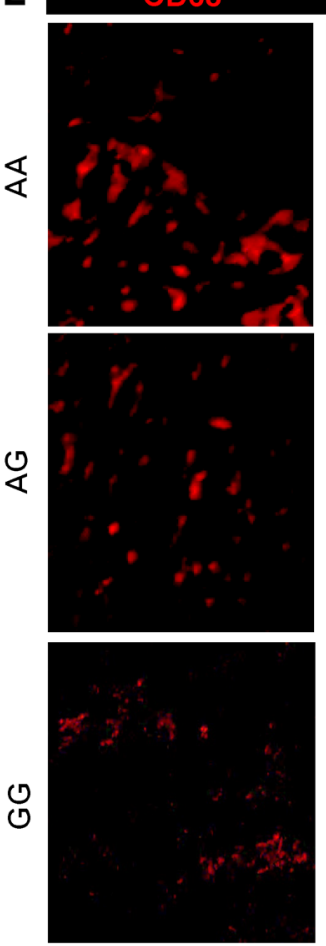

\section{PHACTR1}
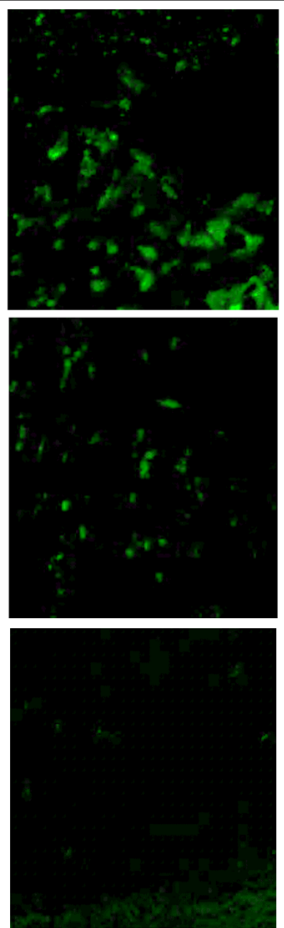

\section{Merged}
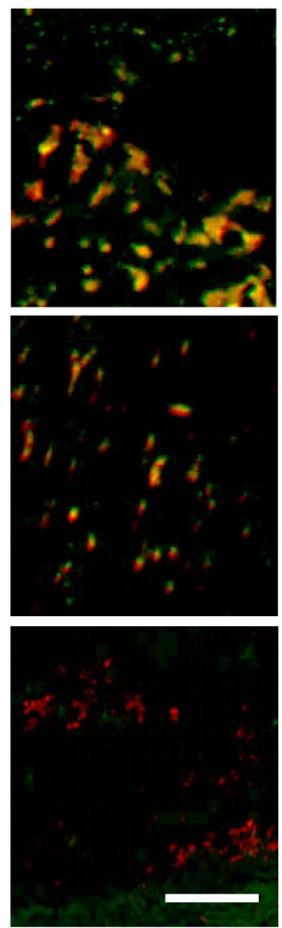

$\mathbf{F}$

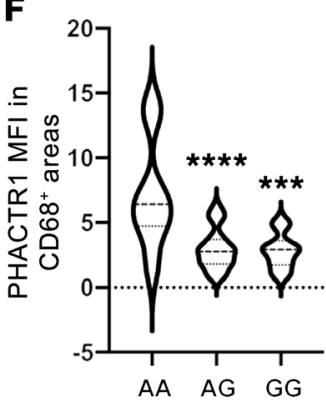

H

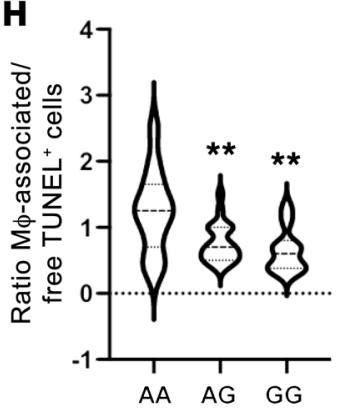

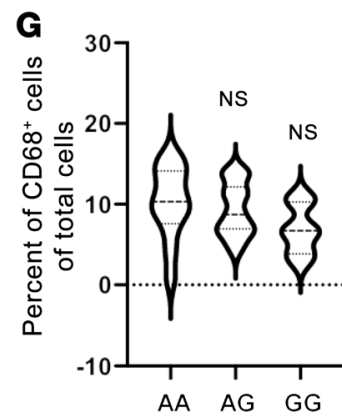

Figure 1. Human monocyte-derived and atherosclerotic lesional macrophages carrying the rs9349379-G CAD-risk variant have lower PHACTR1 expression and impaired efferocytosis. (A-D) Human monocyte-derived macrophages (HMDMs) were genotyped for rs9349379 SNP ( $n=48$; $A A=14, A G=24, G G=10)$ and then assayed as follows: relative PHACTR1 mRNA by quantitative PCR (normalized to HRPT1) (A); PHACTR1 protein ( 75 kDa) by immunoblot (densitometric ratio with GAPDH [36 kDa] and expressed relative to $A A)(B)$; and percentage of HMDMs that engulfed PKH26-labeled $A C s$ (C). (D) Plot of efferocytosis versus PHACTR1 protein expression of rs9349379-AG HMDMs ( $r^{2}$ and $P$ value obtained using Spearman correlation analysis). (E-H) Human carotid endarterectomy specimens were genotyped for rs9349379 SNP ( $n=45 ; A A=13, A G=22, G G=10)$ and then fixed and sectioned for immunofluorescence microscopy. (E) Sections were costained for PHACTR1, CD68, and DAPI. Scale bar: $100 \mu \mathrm{m}$. (F and G) The mean fluorescence intensity (MFI) of PHACTR1 in CD68 ${ }^{+}$areas (F) and the percentage of total DAPI ${ }^{+}$cells that were $\mathrm{CD68}^{+}(\mathbf{G})$ were quantified. For each subject, 5 areas were analyzed, and the MFI value was averaged. Sections stained with isotype control antibodies against PHACTR1 and CD68 showed an absence of signal. (H) Sections were costained with TUNEL and CD68. Each TUNEL ${ }^{+}$cell was determined to be either associated with a macrophage or not ("free"), and the data are presented as the ratio of macrophage-associated to free TUNEL+ cells. In A-C and F-H, ${ }^{*} P<0.05,{ }^{* *} P<0.01,{ }^{* *} P<0.001,{ }^{* * *} P<0.0001$ compared with the AA group, using 1-way ANOVA with Dunnett's multiple-comparison test.

ed mechanism-based evidence that the risk variant is associated with lower expression of PHACTR1 itself in endothelial cells (27). In particular, the variant was shown to interrupt a binding site for a transcription factor, myocyte enhancer factor-2 (MEF2), at the rs9349379 locus. Most importantly, PHACTR1 mRNA, but not EDN1 mRNA, was found to be lower both in human endothelial cells engineered to have the rs9349379 risk variant on an isogenic background and in human coronary arteries from subjects bearing the risk variant $(27,29)$. Analysis of the Genotype-Tissue Expression (GTEx) data set confirmed these expression quan- titative trait loci data for various human arterial tissues $(29,30)$. Further, a recent study showed that PHACTR1 mRNA, but not EDN1 mRNA, is lower in human carotid atheroma than in normal carotid tissue (31). These combined data raise the possibility that the rs9349379-G risk variant may be linked to CAD through the lower expression of PHACTR1, i.e., that PHACTR1 is atheroprotective. However, whether CAD risk is due to lower PHACTR1 in endothelial cells, other cell types relevant to atherosclerosis, or a combination of cell types remains to be determined. The possible role of macrophage PHACTR1 in atherosclerosis was suggested 
by a recent study showing that transplantation of atherosclerosis-prone Apoe $e^{-/-}$mice with Phactr1 ${ }^{-/-}$Apoe $^{-/-}$bone marrow aggravated atherosclerosis (32), but possible links to the human CADrisk polymorphism or efferocytosis remain unknown.

In this study, we investigated the hypothesis that lower expression of PHACTR1 in macrophages in subjects carrying the rs9349379-G allele contributes to atherosclerosis by compromising efferocytosis, specifically the ability of macrophages to internalize bound ACs. PHACTR1 contains 4 highly conserved RPEL domains that interact with actin and protein phosphatase 1 (PP1); and a nuclear localization signal (NLS) that facilitates PHACTR1 nuclear translocation (33-36). In NIH 3T3 fibroblasts, G-actin interaction with the RPEL domains maintains PHACTR1 in the cytoplasm, while serum-induced $\mathrm{G}$ - to F-actin polymerization prevents this binding and thereby promotes the translocation of PHACTR1 into the nucleus (36). This series of events decreases PP1 phosphatase activity in the cytoplasm, leading to increased levels of phosphorylated myosin light chain (MLC) in the cytoplasm and contraction of actinomycin complex (36). Other studies with various non-macrophage cell types have also suggested that PHACTR1 influences actin dynamics and mediates actin-mediated cell motility and membrane protrusions $(37,38)$, but the role of PHACTR1 in efferocytosis remains unknown. By immunohistochemistry, PHACTR1 was found to be highly expressed in macrophages in human atherosclerotic lesions, and the rs9349379 risk variant was associated with lower expression of a particular short PHACTR1 transcript in human macrophages (39). However, the significance of this remains unknown, as this transcript, if translated, would encode a protein lacking important actin-related functional domains of PHACTR1.

Given the role of actin dynamics in efferocytosis (40), we wondered whether lower full-length PHACTR1 protein expression in lesional macrophages of rs9349379-G subjects might contribute to CAD risk by impairing efferocytosis. In support of this hypothesis, we show that both monocyte-derived macrophages and macrophages in atherosclerotic lesions of rs9349379-G/G subjects have lower expression of full-length PHACTR1 mRNA and PHACTR1 protein and impaired AC engulfment in comparison with rs9349379-A/A subjects. Silencing PHACTR1 in human and mouse macrophages also impaired AC engulfment, and Western diet-fed (WD-fed) $\mathrm{Ldlr}^{-/}$mice with transplanted Phactr1 ${ }^{-/-}$or Phac$\operatorname{tr} \mathrm{I}^{+/}$bone marrow had decreased lesional macrophage efferocytosis and increased features of plaque instability, notably increased plaque necrosis, compared with WD-fed $\mathrm{Phactr}^{+/+} \rightarrow \mathrm{Ldlr}^{--}$mice. Mechanistic data showed that the compromise in efferocytosis in Phactr1 ${ }^{-/-}$macrophages is related to defective AC engulfment owing to decreased phospho-MLC secondary to increased cytoplasmic PP1 $\alpha$. These data support the hypothesis that lower expression of PHACTR1 in subjects with the rs9349379 risk allele contributes to atherosclerotic CAD by impairing lesional macrophage efferocytosis and thereby promoting plaque instability.

\section{Results}

Human monocyte-derived and atherosclerotic lesional macrophages carrying the rs9349379-G CAD-risk variant have lower PHACTR1 expression and impaired efferocytosis. To investigate the effect of the rs9349379 risk allele on macrophage PHACTR1 expression, we used human monocyte-derived macrophages (HMDMs) obtained from anonymous New York Blood Center donors. We detected various protein isoforms of PHACTR1 in HMDMs by immunoblotting, including short $(25 \mathrm{kDa})$, intermediate $(\sim 50 \mathrm{kDa})$, and long ( $75 \mathrm{kDa})$ isoforms (Supplemental Figure 1A; supplemental material available online with this article; https://doi.org/10.1172/ JCI145275DS1). While there were no differences in the expression of the short and intermediate isoforms of PHACTR1 between the SNP carriers and noncarriers (Supplemental Figure 1B), there was a lower expression of PHACTR1 mRNA and the full-length isoform of PHACTR1 protein in HMDMs carrying both copies of the CADrisk allele $(\mathrm{GG})$ versus $\mathrm{AA}$ or $\mathrm{AG}$, and there was a trend toward lower PHACTR1 in AG versus AA (Figure 1, A and B). As noted in the Introduction, PHACTR1 encodes an actin-binding protein, which suggests a possible role in efferocytosis, as actin remodeling is a critical step in the internalization of ACs. We hypothesized that lower expression of PHACTR1 in rs9349379-GG HMDMs would lead to impaired efferocytosis, linking this risk allele to an important and established mechanism driving atherosclerosis progression and complications (7-10). Accordingly, the AA, AG, and GG HMDMs were assayed for their ability to engulf PKH26-labeled ACs. The data show that efferocytosis was lower in GG HMDMs than in AA or AG HMDMs, with AG HMDMs showing a trend toward an intermediate value between GG and AA (Figure 1C). As PHACTR1 protein expression in the 24 AG HMDMs showed a high degree of variation, we plotted PHACTR1 expression versus efferocytosis and found a statistically significant correlation (Figure 1D).

As mentioned, PHACTR1 was found to be highly expressed in macrophages in human atherosclerotic lesions by immunohistochemistry (39). To gain further insight into cell-type expression of PHACTR1 in human atheroma, we used single-cell RNA-Seq to examine PHACTR1 expression in multiple cell types from a human atherosclerotic carotid artery, including macrophages and monocytes (Supplemental Figure 2, A and B). PHACTR1 was among the top differentially expressed genes in macrophages (fold change $=$ $1.71, P=3.13 \times 10^{-165}$ ) and monocytes (fold change $=3.36, P=5.22$ $\times 10^{-236}$ ) compared with other cells (Supplemental Figure $2 \mathrm{C}$ ). The macrophage population was characterized by a high expression of FOLR2, which was shown previously to be upregulated in human atherosclerotic plaques (41) (Supplemental Figure 2D). This population corresponds to the "Folr 2 hi macrophages" reported by Lin et al. (42), and these in turn are similar to the "resident-like macrophages" described by Cochain et al., which have a gene profile that overlaps partially with that of resolving-type macrophages and are distinct from 2 other subpopulations of lesional macrophages whose transcript profile suggests an inflammatory phenotype (43). These data raise the possibility that PHACTR1 may be particularly important in lesional macrophages that lie closer to the resolving end rather than the inflammatory end of the macrophage-phenotype spectrum.

As further support of lesional macrophage PHACTR1 expression, we found by immunofluorescence microscopic analysis of human atherosclerotic carotid arteries that most of the PHACTR1 signal colocalized with lesional $\mathrm{CD}^{+} 8^{+}$macrophages (Figure 1E). Most importantly, this signal was lower in GG and AG plaques versus AA plaques (Figure $1 \mathrm{~F}$ ), while there were no significant differences in the total number of macrophages (Figure $1 G$ ). We then assayed these plaques for $\mathrm{TUNEL}^{+}$cells that were either associated with lesional macrophages or unassociated ("free"), the ratio 
$\mathbf{A}$
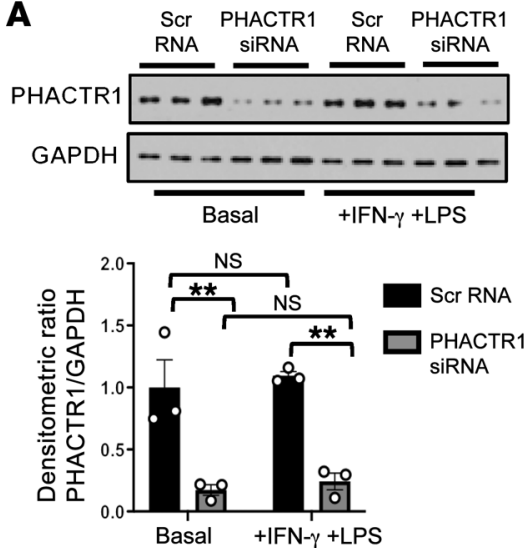

B
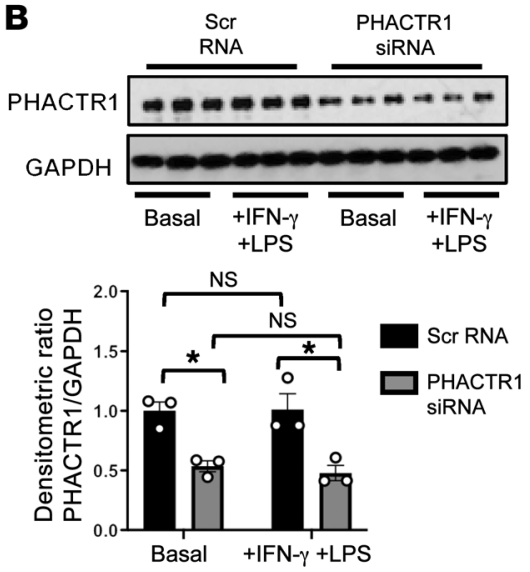

C
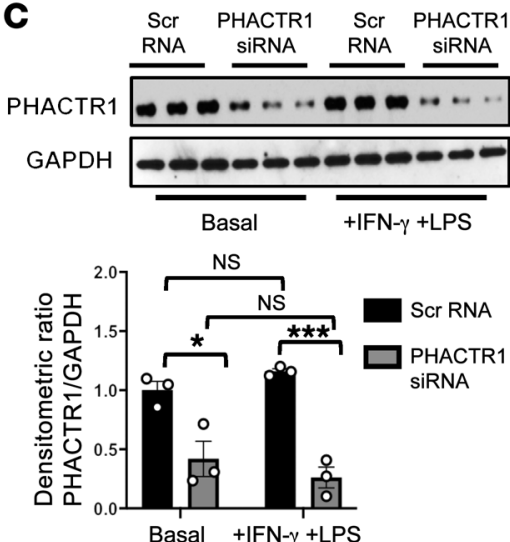
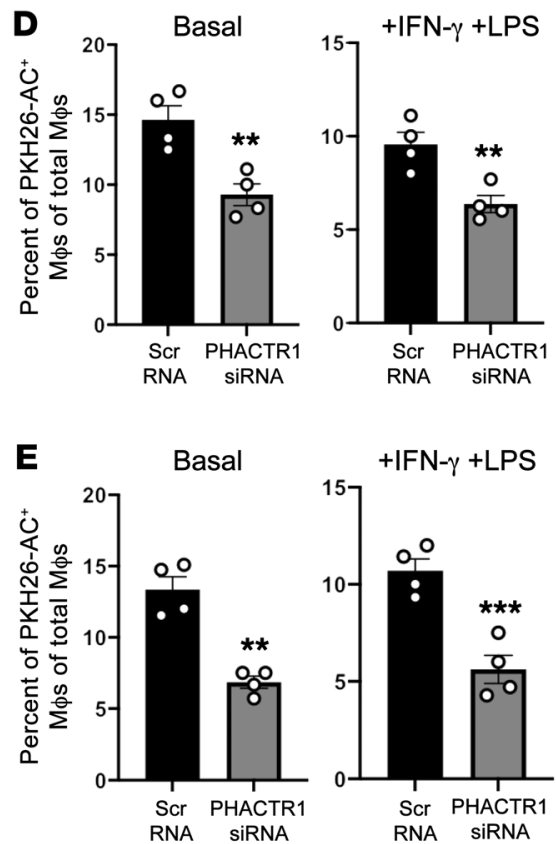

H
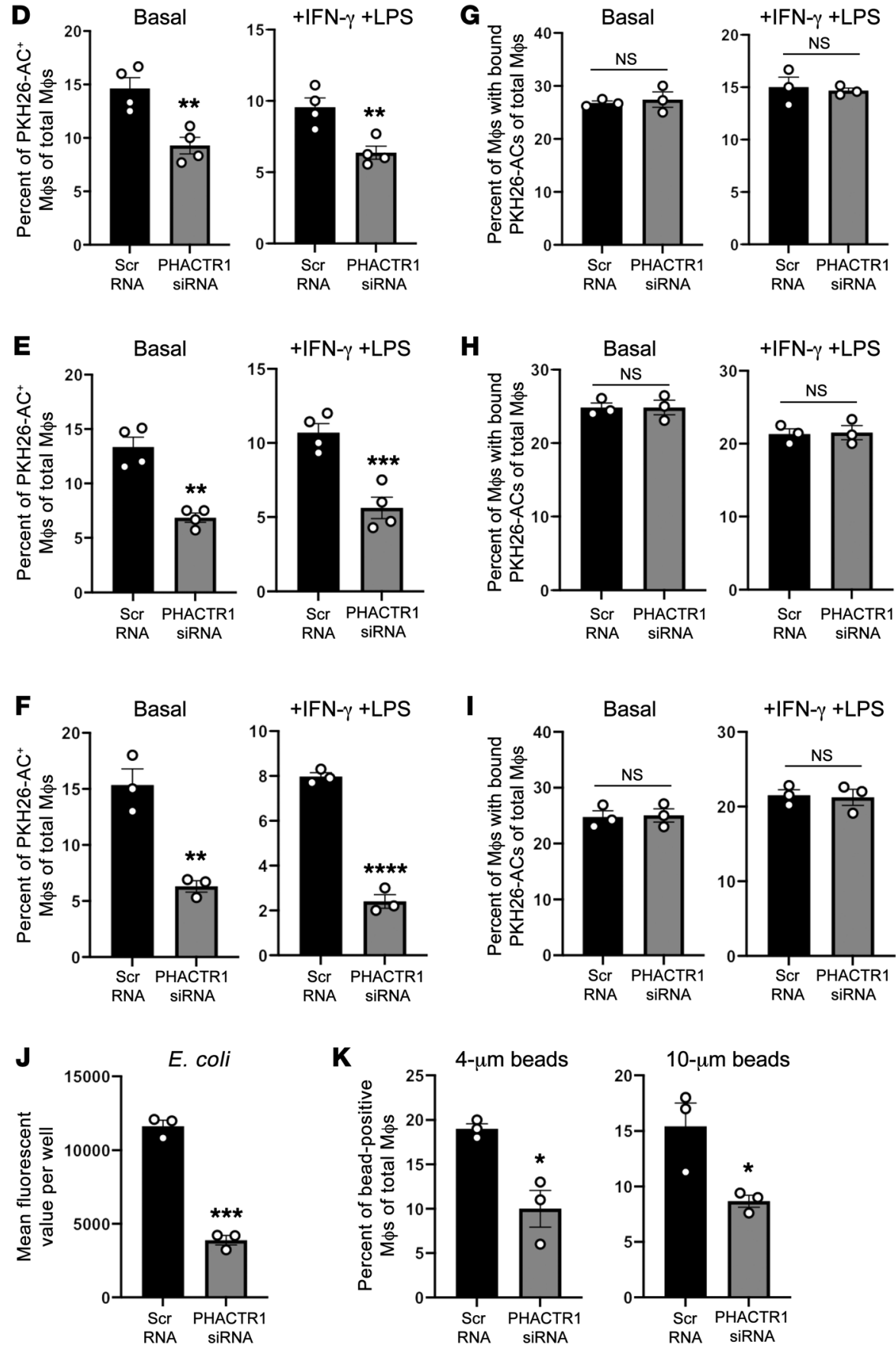

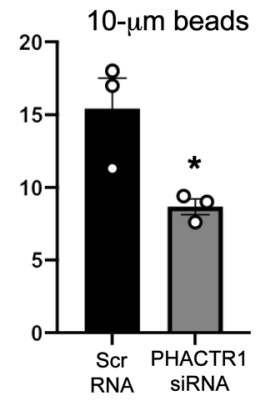

Figure 2. Silencing PHACTR1 in HMDMs decreases efferocytosis and phagocytosis. HMDMs from 3 of the rs9349379-CG subjects in Figure 1 were transfected with either scrambled RNA (Scr RNA) or PHACTR1 siRNA. The cells were then incubated with or without IFN- $\gamma$ and lipopolysaccharide (LPS). (A-C) Immunoblots for PHACTR1 ( 75 kDa) and GAPDH (36 kDa) using HMDMs from subjects 1, 2, and 3, respectively, with quantification of densitometric data. Results are shown as mean $\pm \mathrm{SEM} ; n=3$ experiments; ${ }^{*} P<0.05,{ }^{*} P<0.01,{ }^{* * *} P<0.001$ by 2 -way ANOVA with post hoc Tukey's analysis. (D-F) Efferocytosis of PKH26-labeled ACs by HMDMs from subjects 1, 2, and 3, respectively. (G-I) AC binding to HMDMs from subjects 1, 2, and 3, respectively, pretreated with $5 \mu \mathrm{M}$ cytochalasin D for 20 minutes. (J and $\mathbf{K}$ ) Internalization of $E$. coli and $4-\mu \mathrm{m}$ and $10-\mu \mathrm{m}$ polystyrene beads by HMDMs from subject 1 . For $\mathbf{D}-\mathbf{K}$, $n=3$ biological replicates, using the average of technical triplicates for each. Results are shown as mean \pm SEM, including individual data points. ${ }^{*} P<0.05$, ${ }^{* *} P<0.01,{ }^{* * *} P<0.001,{ }^{* * *} P<0.0001$ by unpaired 2 -tailed Student's $t$ test.

of which is a reliable measure of efferocytosis in tissue specimens (44). The data show that plaques from subjects carrying one or both rs9349379-G alleles had a lower associated/free ratio, indicating relatively impaired efferocytosis (Figure $1 \mathrm{H}$ ). In summary, the rs9349379-G variant, which confers higher CAD risk, tracks with lower levels of macrophage PHACTR1 and impaired efferocytosis both in vitro and in human atherosclerotic lesions.

Silencing PHACTR1 in HMDMs decreases efferocytosis and phagocytosis. To show causation between PHACTR1 and efferocytosis, HMDMs from 3 of the rs9349379-AA subjects in Figure 1 were 
A

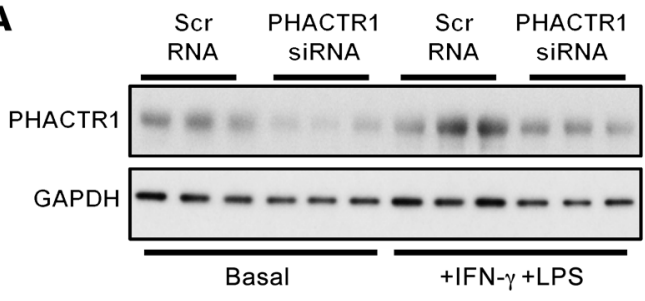

B

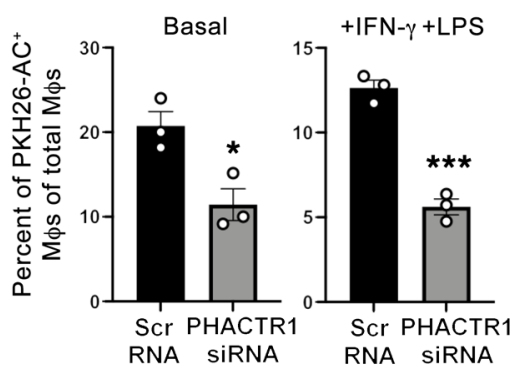

C
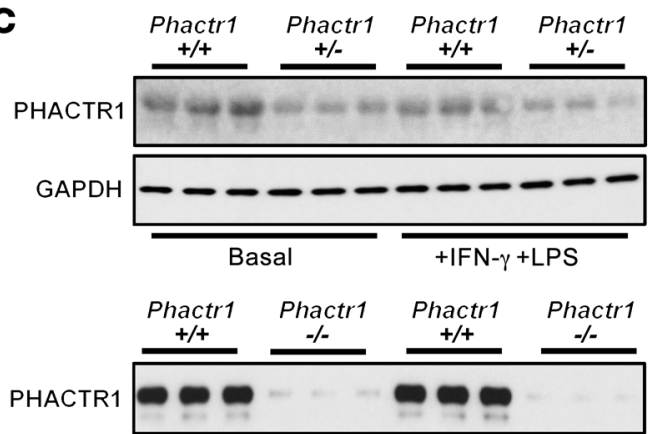

GAPDH

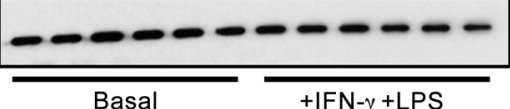

D

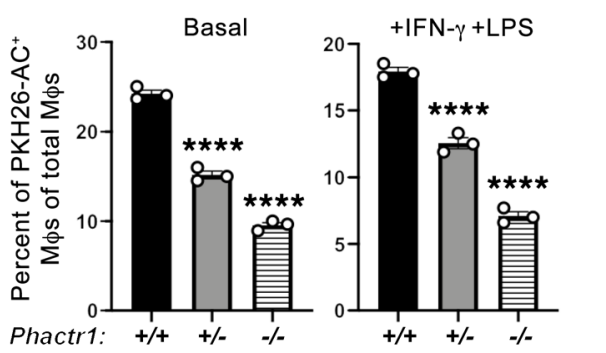

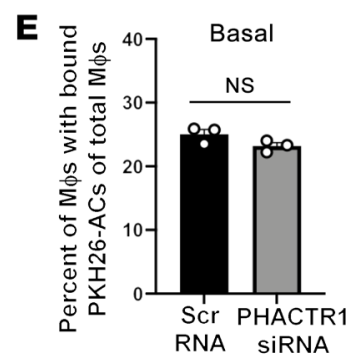
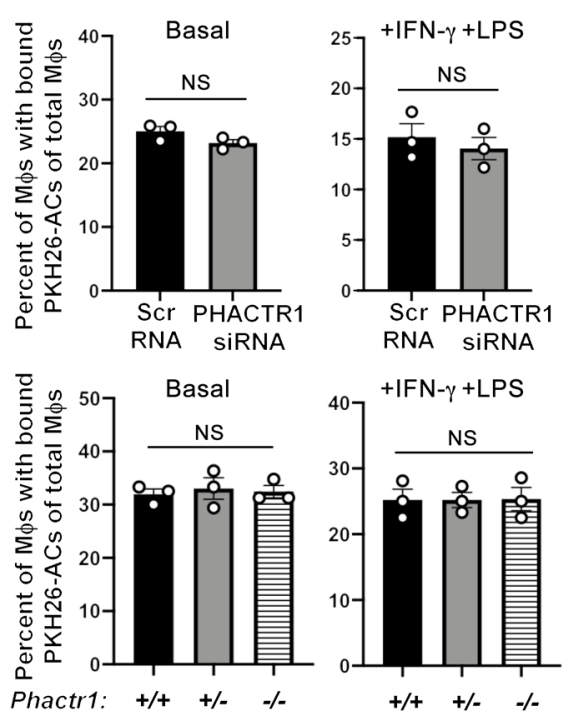

$\mathbf{F}$
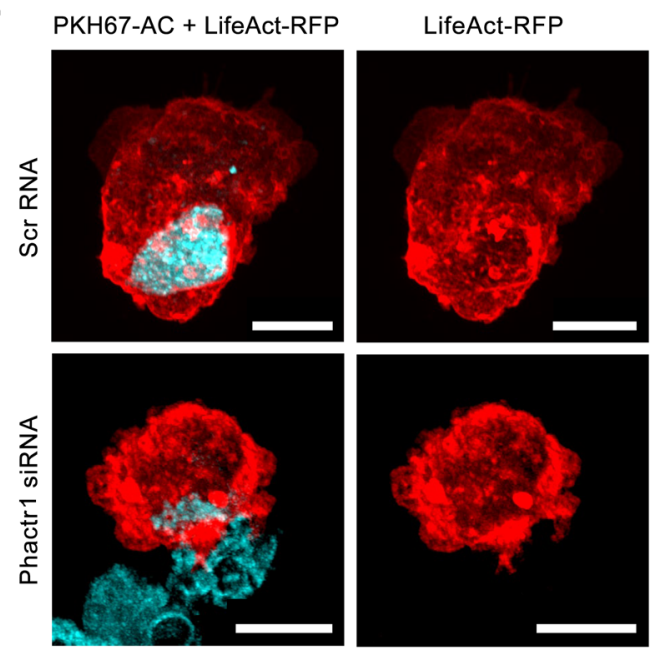

Figure 3. PHACTR1 deficiency impairs phagocytic internalization of ACs in BMDMs. (A-D) Bone marrow-derived macrophages (BMDMs) transfected with either scrambled RNA (Scr RNA) or Phactr1 siRNA (A and B), or from Phactr1 ${ }^{+/+}$, Phactr1 ${ }^{+/-}$, or Phactr1 ${ }^{-/-}$mice (C and D), were incubated with or without IFN- $\gamma$ and LPS and then assayed for PHACTR1 protein ( $72 \mathrm{kDa}$ ) by immunoblot or for efferocytosis. (E) BMDMs from A-D were assayed for AC binding in the presence of cytochalasin D. (F) BMDMs treated with Scr RNA or Phactr1 siRNA were transfected with LifeAct-RFP and then incubated with PKH67labeled ACs for 45 minutes. After fixation, the cells were viewed by confocal fluorescence microscopy. Two examples of 0.5 - $\mu$ m $Z$-stack images from each

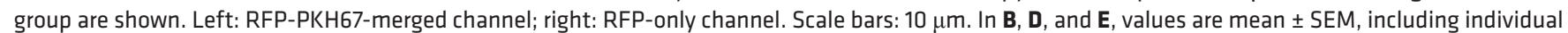
data points; $n=3$ experiments. In $\mathbf{B}$ and upper $\mathbf{E},{ }^{*} P<0.05,{ }^{* *} P<0.001$ by unpaired 2 -tailed Student's $t$ test. In $\mathbf{D}$ and lower $\mathbf{E}$, ${ }^{* * *} P<0.0001$ compared

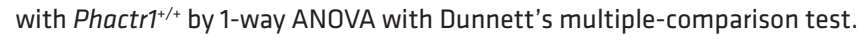

pretreated with either scrambled RNA or siPHACTR1. The cells were incubated with or without IFN- $\gamma$ and lipopolysaccharide (LPS) to model inflammatory macrophages in atherosclerosis and then assayed for efferocytosis as in Figure 1C. IFN- $\gamma /$ LPS treatment did not change PHACTR1 expression, and we achieved good silencing with siPHACTR1 (Figure 2, A-C). For all 3 sets of HMDMs under both basal and inflammatory conditions, PHACTR1 silencing led to impaired efferocytosis (Figure 2, D-F). The impairment was due to lower AC internalization, as siPHACTR1 did not lower AC binding to the macrophages (Figure 2, G-I). To determine whether the defect in $\mathrm{AC}$ internalization was reflecting a general defect in phagocytosis, we tested the ability of the macrophages to inter- 
A
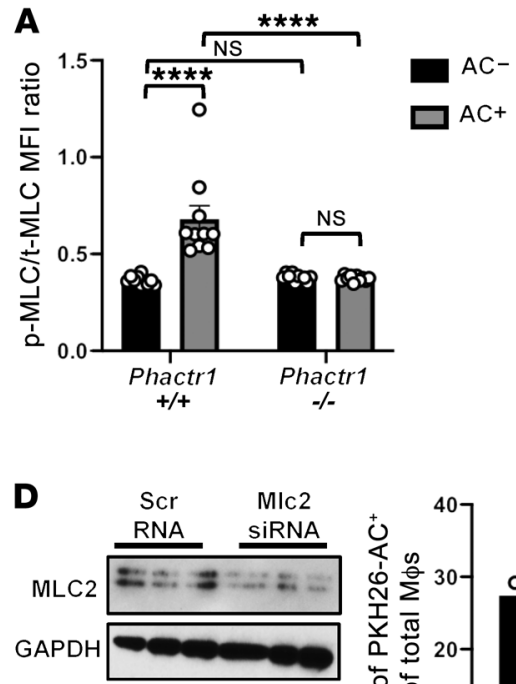
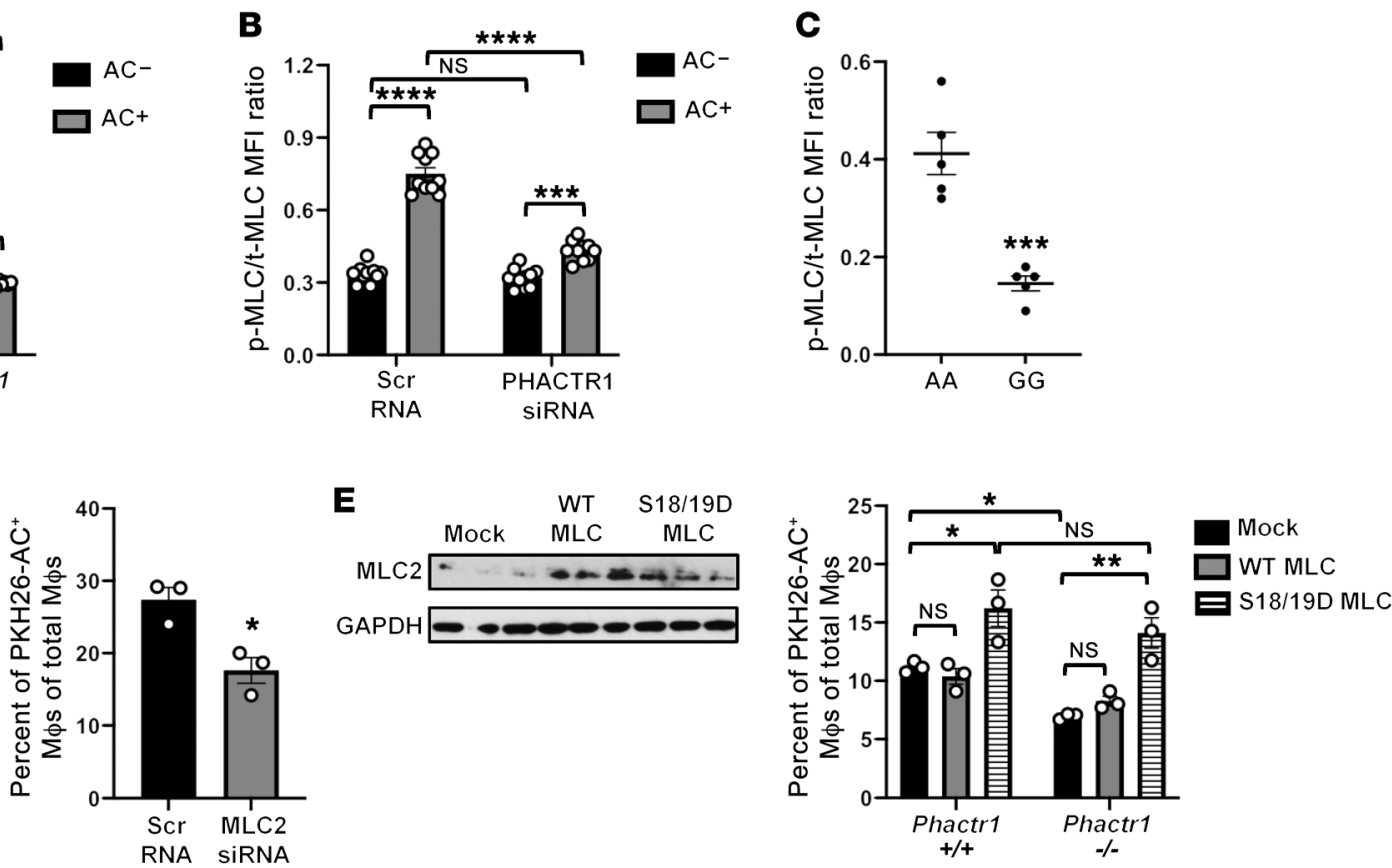

Figure 4. PHACTR1 facilitates efferocytosis by increasing MLC phosphorylation. (A) Ratio of MFI of phospho-MLC (p-MLC) to total MLC (t-MLC) was quantified in Phactr1+/+ and Phactr1 ${ }^{-/-}$BMDMs incubated in the absence or presence of ACs (AC-, AC+). (B) HMDMs were treated with scrambled RNA or PHACTR1 siRNA and assayed for $\mathrm{p}-\mathrm{MLC} / \mathrm{t}-\mathrm{MLC}$ MFI ratio as in A. ${ }^{* * *} P<0.001$, ${ }^{* * *} P<0.0001$. (C) Ratio of $\mathrm{p}-\mathrm{MLC}$ to $\mathrm{t}-\mathrm{MLC}$ was quantified for MFI in $A C+$ rs9349379-GG and -AA HMDMs as in Figure 1. Images were quantified for $p-M L C / t-M L C$ MFI ratio; $n=5$ HMDMs per group; ${ }^{* * *} P<0.001$ by Student's unpaired $t$ test. (D) BMDMs treated with scrambled RNA or MIc2 siRNA were then immunoblotted for MLC (18 kDa) and GAPDH (36 kDa) or assayed for efferocytosis. Results are shown as mean \pm SEM; $n=3$ experiments; ${ }^{*} P<0.05$ by 2-tailed Student's unpaired $t$ test. (E) Phactr ${ }^{1 /+}$ and $P$ Phactr ${ }^{-/-}$BMDMs were transfected with empty vector (Mock) or vector encoding WT MLC or S18/19D MLC. One set of cells was immunoblotted for MLC and GAPDH, and the other was assayed for efferocytosis. Results are shown as mean $\pm \mathrm{SEM}$, including individual data points; $n=3$ experiments; ${ }^{*} P<0.05$, ${ }^{* *} P<0.01$ by 2 -way ANOVA with post hoc Tukey's analysis.

nalize E. coli and 4- $\mu \mathrm{m}$ and $10-\mu \mathrm{m}$ polystyrene beads. For all 3 substrates, siPHACTR1 lowered uptake by the macrophages (Figure 2, J and K). Thus, silencing PHACTR1 in HMDMs causes a general defect in phagocytosis, including a defect in efferocytosis.

In anticipation of the in vivo molecular and genetic causation studies in mice, described below, we assayed the role of PHACTR1 in efferocytosis in murine bone marrow-derived macrophages (BMDMs) from Phactr1 ${ }^{+/+}$, Phactr1 ${ }^{+/}$, and Phactr1 ${ }^{1 /-}$ mice. As with PHACTR1-silenced HMDMs, basal and IFN- $\gamma /$ LPS-treated BMDMs from Phactr1 ${ }^{+/}$mice showed decreased efferocytosis compared with Phactr1 ${ }^{+/+}$BMDMs (Figure 3, A and B), and this impairment was enhanced in Phactr1 ${ }^{-/}$BMDMs (Figure 3, C and D). Also similar to the results with HMDMs, PHACTR1 silencing or genetic targeting did not block AC binding to mouse BMDMs (Figure 3E). Finally, given the defect in AC internalization and the role of PHACTR1 as an actin-binding protein, we reasoned that PHACTR1 deficiency might alter actin remodeling around the phagocytic cup that forms around a bound AC. In support of this idea, we found that PHACTR1 silencing in BMDMs labeled with the F-actin marker LifeAct-RFP caused a defect in the formation of the typical wide-mouth actin cup around an engaged AC (Figure 3F). The uptake of oxidized LDL, which occurs through a mechanism independent of the actin cytoskeleton (45), was not different between PhactrI $^{+/+}$and Phactr1 ${ }^{-/}$BMDMs (Supplemental Figure 3). In summary, silencing PHACTR1 in human and mouse macrophages causes a specific defect in the phagocytic internalization of ACs.
PHACTR1 facilitates efferocytosis by decreasing PP1a-mediated MLC dephosphorylation. PHACTR1 mediates the assembly of stress fibers in fibroblasts by maintaining the phosphorylated, active form of myosin light chain (MLC), which facilitates actomyosin contraction (36). Given the role of actin in AC engulfment, we hypothesized that PHACTR1 enables efferocytosis by a similar mechanism. Consistent with this hypothesis, we showed that the binding of ACs to BMDMs and HMDMs induces phosphorylation of MLC in a PHACTR1dependent manner (Figure 4, A and B). Moreover, rs9349379-GG HMDMs, which have lower PHACTR1 than rs9349379-AA HMDMs, also had lower phospho-MLC (Figure 4C). Most importantly, silencing of MLC blocked efferocytosis in WT macrophages, and transfection with constitutively activated $\mathrm{Ser}^{18,19} \rightarrow \mathrm{Asp}^{18,19}$ MLC restored efferocytosis in PHACTR1-deficient macrophages (Figure 4, D and E).

MLC, a cytoplasmic protein, can be dephosphorylated by cytoplasmic PP1 $\alpha$ but not by nuclear PP1 $\alpha$ (46-48). In context, there is evidence in serum-stimulated fibroblasts to suggest that PHACTR1 can bind PP1 $\alpha$ and facilitate its translocation to the nucleus in a process dependent on a nuclear localization signal (NLS) in PHACTR1 (36). To test the relevance of this pathway to macrophages carrying out efferocytosis, we conducted a series of experiments in which various factors in the pathway were genetically manipulated. First, exposure of BMDMs to ACs increased the binding of PHACTR1 to PP1 $\alpha$, as assessed by immunoblotting for PHACTR1 in anti-PP1 $\alpha$ immunoprecipitates (Figure 5A). Second, PP1 $\alpha$ silencing increased the ratio of phospho- to total 
A

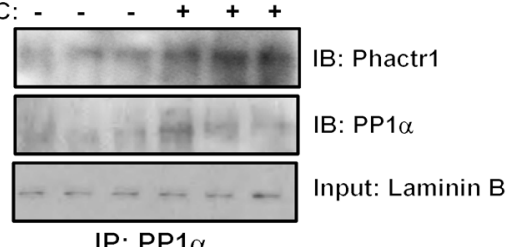

C

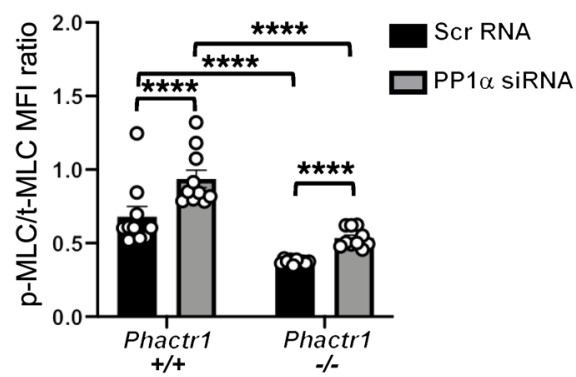

E
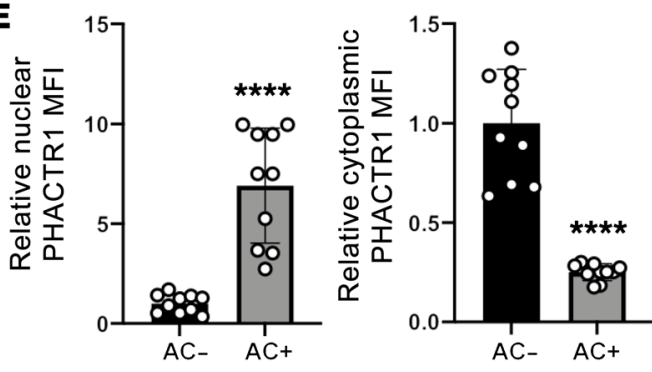

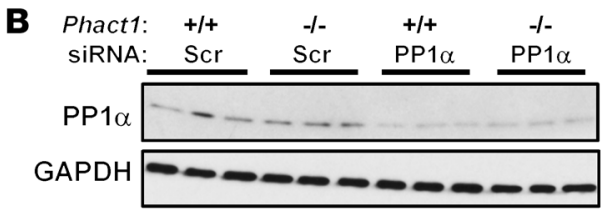

D

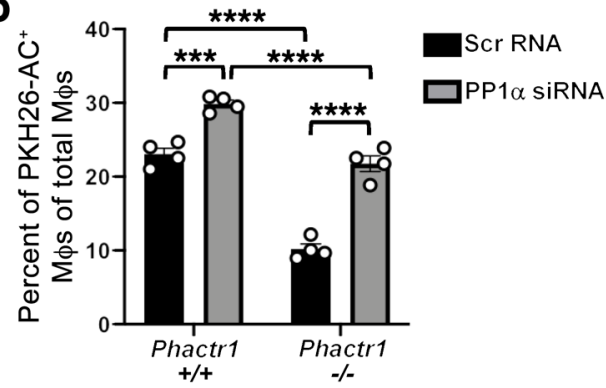

$\mathbf{F}$
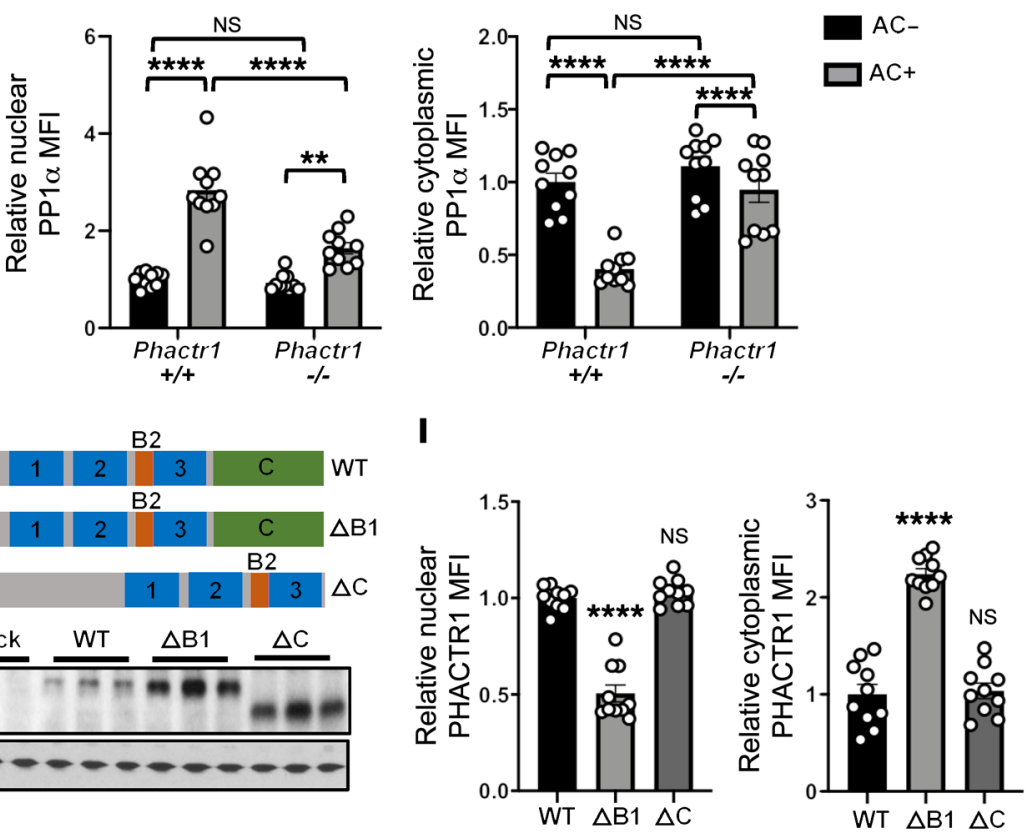
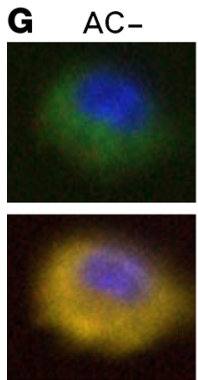

Phactr1 PP1 $\alpha$ AC DAPI (nuclei)
H

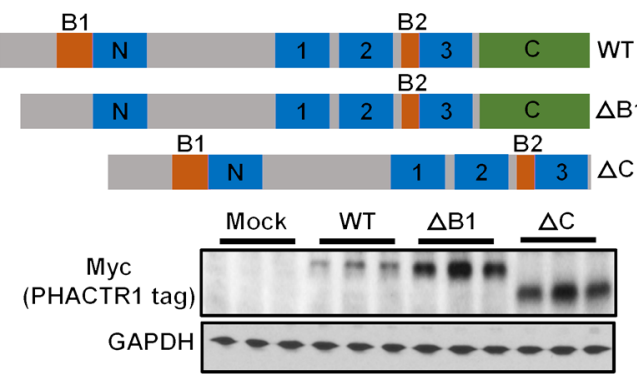

I
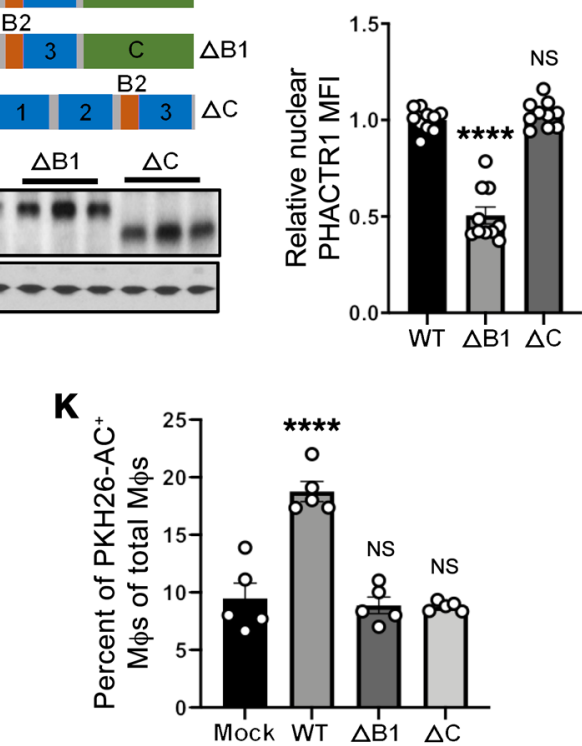
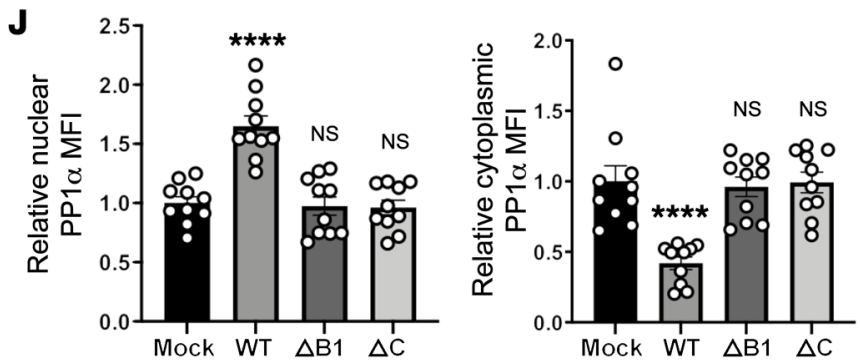

Figure 5. PHACTR1 sequesters PP1 $\alpha$ in the nucleus and decreases the dephosphorylation of MLC during efferocytosis. (A) Immunoblot of PHACTR1 ( 72 $\mathrm{kDa}$ ), PP1 $\alpha$ (38 kDa), and laminin B (input control; $68 \mathrm{kDa}$ ) of anti-PP1 $\alpha$ immunoprecipitates from BMDMs incubated in the absence or presence of ACs. (B-D) Phactr1+/+ and Phactr1//- BMDMs treated with scrambled RNA or PP1 $\alpha$ siRNA. One set of cells was immunoblotted for PPA1 $\alpha$ (38 kDa) and GAPDH (36 $\mathrm{kDa}$ ), one set was stained with phospho- and total MLC antibody and quantified as phospho- to total MLC MFI ratio, and one set was assayed for efferocytosis. (E) Quantification of nuclear and cytoplasmic PHACTR1 in BMDMs incubated in the absence or presence of ACs. (F) Quantification of nuclear and cytoplasmic PP1 $\alpha$ in Phactr1 ${ }^{+/+}$and Phactr1 ${ }^{-/-}$BMDMs incubated in the absence or presence of ACs. (C) Immunofluorescence microscopy images of BMDMs incubated in the absence or presence of ACs (green, PHACTR; yellow, PP1 $\alpha$; red, AC; blue, DAPI [nucleus]). (H) Graphic scheme of PHACTR1 WT and mutant protein structure and immunoblots of Myc (PHACTR1 tag) and GAPDH in Phactr1 ${ }^{-/-}$BMDMs transfected with WT and mutant PHACTR1 (Myc-tagged PHACTR1 protein is $73 \mathrm{kDa}$ in WT and $\triangle \mathrm{B} 1$, and $70 \mathrm{kDa}$ in $\Delta \mathrm{C})$. (I-K) Quantification of nuclear and cytoplasmic PHACTR1 and PP1 $\alpha$ and efferocytosis in the BMDMs depicted in $\mathbf{H}$. In C-F and I-K, mean \pm SEM, including individual data points; $n=3$ experiments, with $n>5-10$ macrophages quantified for each group; ${ }^{* *} P<0.01,{ }^{* *} P<0.001,{ }^{* * *} P<0.0001$ by 2 -tailed Student's unpaired $t$ test (C-F), 1-way ANOVA with post hoc Dunnet's analysis compared with WT (I), or 2-way ANOVA with post hoc Tukey's analysis, compared with mock (J and $\mathbf{K})$. 
A

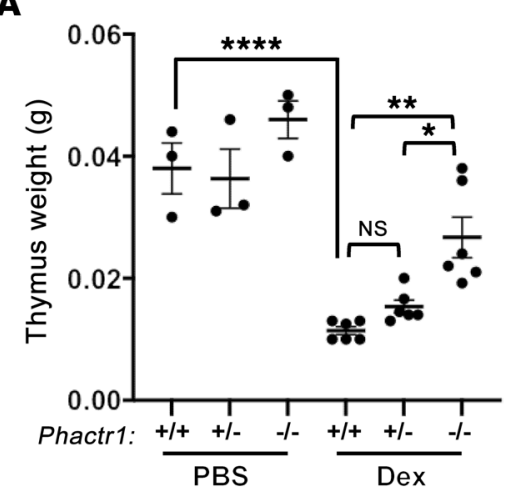

D

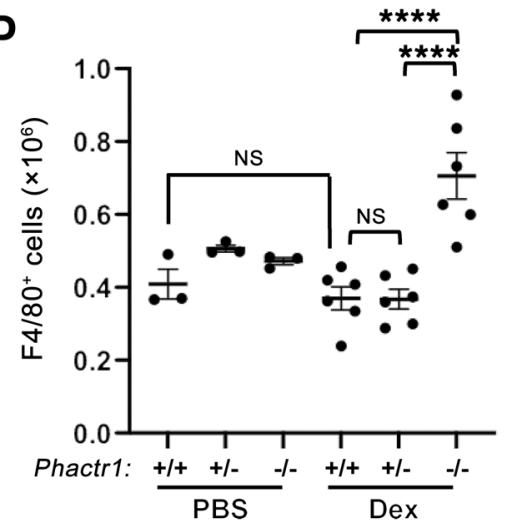

B

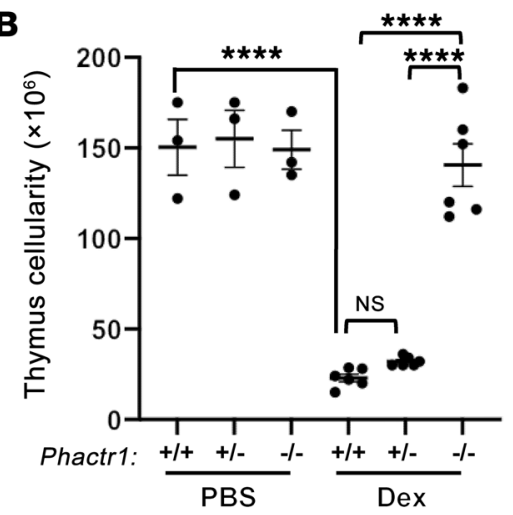

E

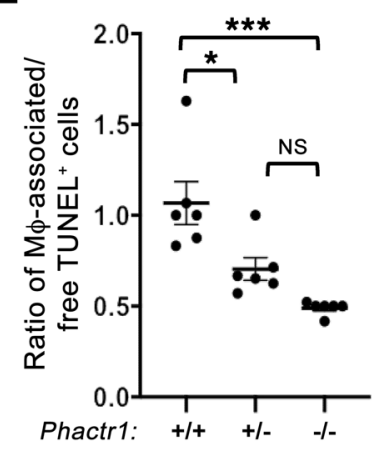

C

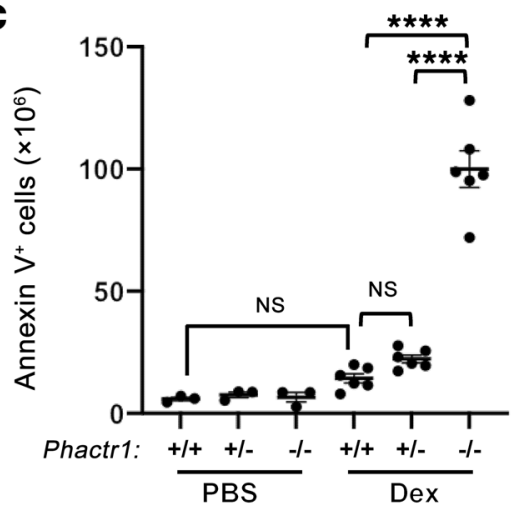

$\mathbf{F}$

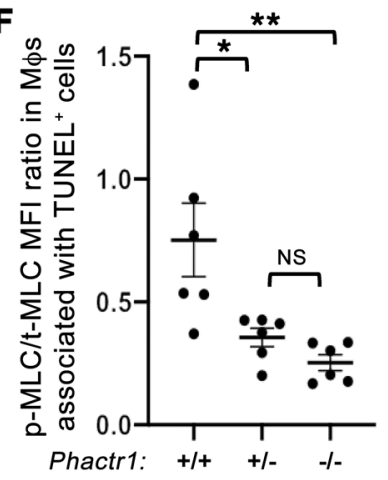

Figure 6. Chimeric mice lacking hematopoietic PHACTR1 show defective macrophage efferocytosis of apoptotic thymocytes. Bone marrow cells from Phactr $1^{+/+}$, Phactr $7^{+/}$, or Phactr1 $7^{-/-}$mice were transplanted into irradiated mice. After 6 weeks, the mice were injected i.p. with PBS or $250 \mathrm{mg} / \mathrm{mouse}$ dexamethasone. After 18 hours, the thymi were harvested. (A-D) Thymic weight, cellularity, and content of annexin $\mathrm{V}^{+}$cells and $\mathrm{F} 4 / 80^{+}$cells. (E) Thymus sections were stained for TUNEL and Mac2 and then quantified for the ratio of macrophage-associated to free TUNEL+ cells. (F) Thymus sections were stained for TUNEL, Mac2, p-MLC, and t-MLC, and the ratio of p-MLC to t-MLC was quantified for MFI in macrophages associated with TUNEL ${ }^{+}$cells. In A-F, results are shown as individual data points with lines indicating mean $\pm S E M ; n=3$ mice for PBS groups and $n=6$ mice for dexamethasone groups; ${ }^{*} P<$ $0.05,{ }^{* *} P<0.01,{ }^{* *} P<0.001,{ }^{* * * *} P<0.0001$ by 1 -way ANOVA with Dunnett's multiple-comparison test.

MLC in both WT and Phactr1-/- BMDMs and, most importantly, normalized efferocytosis in Phactr1 ${ }^{-/}$macrophages (Figure 5 , B-D). Third, exposure of BMDMs to ACs increased nuclear PHACTR1 and PP1 $\alpha$ and decreased cytoplasmic PHACTR1 and PP1 $\alpha$ as assessed by both immunoblot and immunofluorescence microscopy (Figure 5, E-G). Fourth, we studied Phactr1 ${ }^{-/}$BMDMs reconstituted with Myc-tagged WT PHACTR1 or PHACTR1 lacking either its NLS domain $(\Delta \mathrm{B} 1)$ or PP1 $\alpha$-binding domain $(\Delta \mathrm{C})$ (Figure $5 \mathrm{H}$ ). As expected, AC-exposed Phactr1 ${ }^{-/}$macrophages transfected with the NLS-lacking B1 PHACTR1 mutant showed a markedly lower nuclear/cytoplasmic ratio of PHACTR1 compared with cells reconstituted with WT PHACTR1 (Figure 5I). Moreover, reconstitution of Phactr1-- macrophages with either of the mutant PHACTR1 forms led to a lower nuclear/cytoplasmic ratio of PP1 $\alpha$ compared with cells reconstituted with WT PHACTR1 (Figure 5J), supporting the role of macrophage PHACTR1 in facilitating the translocation of PP1 $\alpha$ to the nucleus. Most importantly, while reconstitution with WT PHACRT1 rescued the efferocytosis defects in Phactr1 ${ }^{-/}$BMDMs, reconstitution with the mutant PHACTR1 forms lacking the NLS domain or PP1 $\alpha$-binding domain failed to rescue the defective efferocytosis in Phactr $1^{-/-}$BMDMs (Figure 5K). These combined data support the hypothesis that PHACTR1 enhances MLC-mediated efferocytosis by sequestering PP1 $\alpha$ in the nucleus and thereby preventing the dephosphorylation-deactivation of cytoplasmic MLC.

Chimeric mice lacking hematopoietic PHACTR1 show defective macrophage efferocytosis of apoptotic thymocytes. To explore the role of macrophage PHACTR1 in efferocytosis in vivo, we investigated efferocytosis in the thymi of dexamethasone-treated mice reconstituted with Phactr1 ${ }^{+/+}$, Phactr1 ${ }^{+/-}$, or Phactr1 1/- bone marrow. Dexamethasone administration causes robust thymocyte apoptosis, followed by AC clearance by thymic macrophages (49). As expected, treatment of control Phactr1 ${ }^{+/+}$-transplanted mice with dexamethasone caused a marked reduction in thymic weight and cellularity (Figure 6, A and B), which is caused by the coupled processes of thymocyte apoptosis and efferocytic disposal of the dead cells. Accordingly, the number of annexin $\mathrm{V}^{+}$(apoptotic) cells in the thymus of these control mice showed only a slight increase (Figure 6C). In contrast, there was much less of a decrease in thymus weight and cellularity and a much greater increase in annexin $\mathrm{V}^{+}$ and F4 $/ 80^{+}$macrophages in Phactr1 ${ }^{-/}$-transplanted mice (Figure 6, C and D). When compared with the Phact1 ${ }^{+/+}$cohort, Phactr1 ${ }^{+/-}$ thymi showed slight trends toward higher thymus weight, cellularity, and ACs, but these differences did not reach statistical sig- 
A

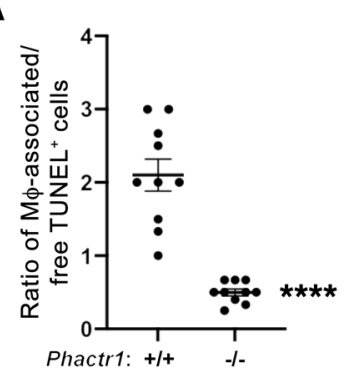

B

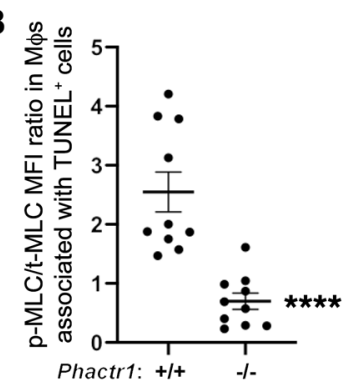

C

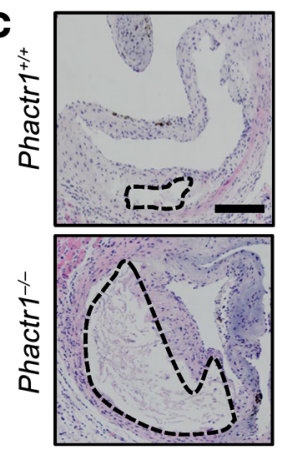

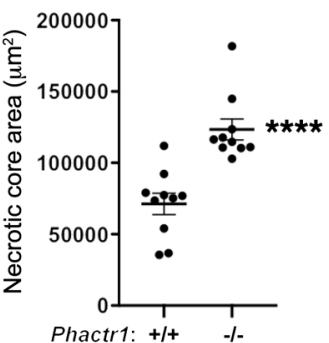

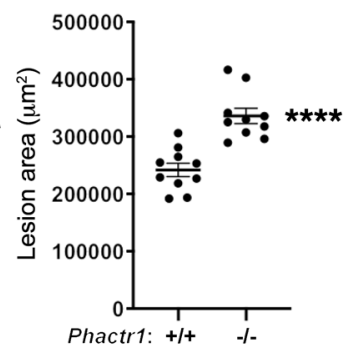

D

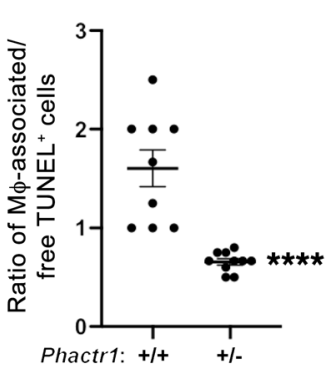

$\mathbf{E}$

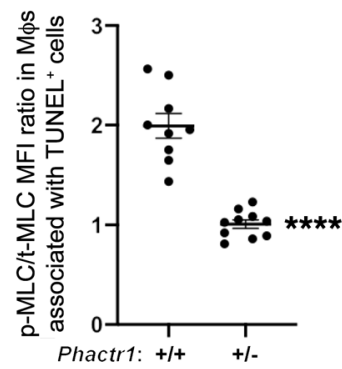

$\mathbf{F}$

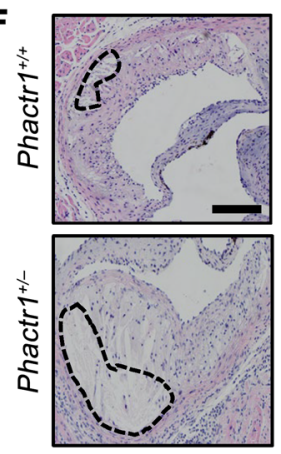

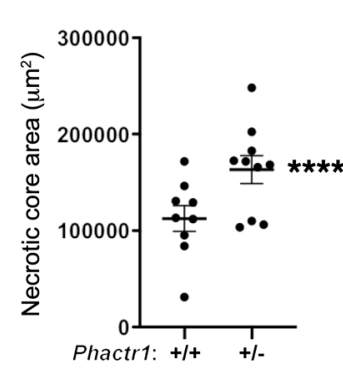

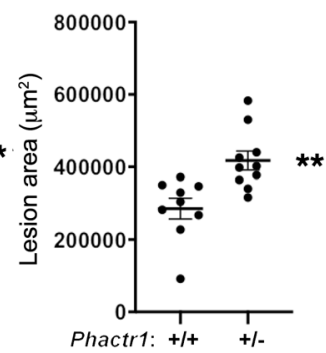

G

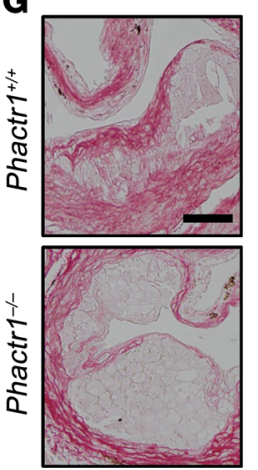

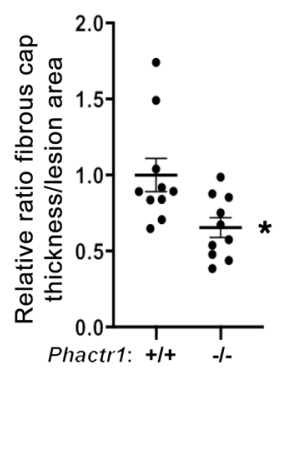
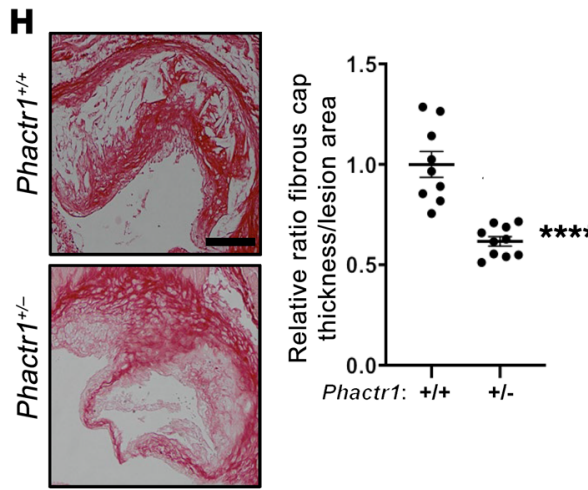

I
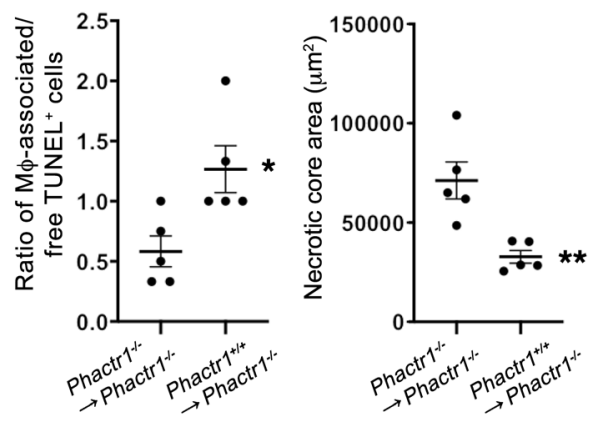

Figure 7. WD-fed LdIr/- mice lacking hematopoietic PHACTR1 show defective macrophage efferocytosis and increased plaque necrosis in atherosclerotic lesions. (A-C and G) Bone marrow from Phactr ${ }^{T^{++}}$or Phactr ${ }^{-/-}$mice was transplanted into irradiated $\mathrm{Ldll}^{-1-}$ mice. After 4 weeks, the mice were fed a Western-type diet (WD) for 8 weeks, and then aortic root lesional cross sections were analyzed. (A) Ratio of Mac2 ${ }^{+}$macrophage-associated to free TUNEL ${ }^{+}$cells. (B) Ratio of p-MLC to t-MLC MFI in macrophages associated with TUNEL+ cells. (C) Images of aortic root sections stained with H\&E (dashed lines indicate necrotic area), with quantification of necrotic and lesion areas. (D-F and H) Bone marrow from Phactr $7^{{ }^{+/}}$or Phactr $7^{+/-}$mice was transplanted into irradiated $L d l r^{1-}$ mice. After 4 weeks, the mice were fed the WD for 12 weeks. (D-F) Lesional endpoints were quantified as above. (G and $\mathbf{H})$ Aortic root cross sections were stained with Picrosirius red. For each section, cap thickness was measured at the lesional midpoint and both shoulder regions and then averaged and quantified as the ratio of collagen cap thickness to lesion area, relative to the Phactr1 $7^{+/}$group. (I) Bone marrow from Phactr1 ${ }^{-/-}$or Phactr 1/+ $^{+/}$mice was transplanted into irradiated Phac$\mathrm{tr}^{-1-} \mathrm{Ldll}^{-1-}$ mice. After 4 weeks, the mice were fed the WD diet for 8 weeks, and lesional endpoints were quantified as above. In all panels, results are shown as individual data points with lines indicating mean $\pm \mathrm{SEM}$; ${ }^{*} P<0.05,{ }^{* *} P<0.01,{ }^{* * *} P<0.0001$ by 2-tailed Student's unpaired $t$ test. Scale bars: $100 \mu \mathrm{m}$.

nificance. Most importantly, the ratio of macrophage-associated to free TUNEL ${ }^{+}$cells, i.e., efferocytosis, was lower in mice reconstituted with $\mathrm{Phactr}^{+/-}$and Phactr1 ${ }^{-/}$bone marrow (Figure 6E). To link these data to our mechanistic work, we assayed the ratio of phospho- to total MLC by immunofluorescence microscopy in AC-associated macrophages in the thymi. We found that most of the positive signal for phospho-MLC was in macrophages associated with TUNEL ${ }^{+}$cells and that, as predicted by our in vitro mechanistic data, the ratio among the 3 cohorts followed the same pattern as that seen with efferocytosis (Figure 6F). These data provide evidence that PHACTR1 is necessary for maintaining WT levels of phospho-MLC and, most importantly, for enabling efficient efferocytosis in macrophages faced with a major AC challenge in vivo.

WD-fed Ldlr- mice lacking hematopoietic PHACTR1 show defective macrophage efferocytosis and increased plaque necrosis in atherosclerotic lesions. Efferocytosis is a critical process in atherosclerosis, as efficient clearance of ACs in atherosclerotic lesions is needed to prevent the accumulation of inflammatory necrotic cells and to promote plaque-stabilizing pro-resolution processes $(7,8)$. Thus, as posited, defective efferocytosis could contribute 
to higher CAD risk in subjects carrying loss-of-expression polymorphisms in PHACTR1. To test this idea in experimental atherosclerosis, bone marrow cells from $\mathrm{Phactrl}^{+/+}, \mathrm{Phactr1}^{+/}$, or Phac$\mathrm{trl}^{\mathrm{I}^{-/}}$were transplanted into lethally irradiated $\mathrm{Ldlr}^{-/-}$mice, which were then placed on a Western-type diet for 8 weeks (Phactr1 ${ }^{-/}$vs. Phactr $^{+/+}$) or 12 weeks (Phactr1 ${ }^{+/-}$vs. Phactrl ${ }^{+/+}$). Immunostaining showed that the macrophage-rich regions of the Phactr $^{+/+}$lesions expressed PHACTR1, which was less in Phactr1 $^{+/}$lesions and undetectable in Phactr1 $^{-/}$lesions (Supplemental Figure 4A). There were no significant differences in body weight, plasma cholesterol, blood glucose, plasma triglycerides, plasma lipoprotein-cholesterol profile, or blood monocytes between control and hematopoietic PHACTR1-deficient mice in either 8-week or 12-week experiments (Supplemental Figure 4, B-M, respectively). In both experiments, macrophage efferocytosis in aortic root atherosclerotic lesions was lower in hematopoietic PHACTR1-deficient mice compared with control mice (Figure 7, A and D), and this was associated with a lower phospho-MLC/total MLC ratio in lesional macrophages that were associated with $\mathrm{TUNEL}^{+}$cells (Figure 7, B and E). Hematopoietic PHACTR1-deficient mice in both experiments showed increases in necrotic core and lesion area (Figure 7, C and F), and decreases in fibrous cap thickness (Figure 7, G and $\mathrm{H}$ ), which is consistent with impaired resolution. In humans, plaque necrosis and thin fibrous caps are associated with plaque instability (11-13). Finally, transplantation of bone marrow from Phactrl $^{+/+}$mice into Phactrl ${ }^{-/-} \mathrm{Ldlr}^{-/-}$recipients $\left(\mathrm{Phactr}^{+/+} \rightarrow \mathrm{Phac}^{-}\right.$ $\mathrm{trI}^{-/-} \mathrm{Ldll}^{-/-}$mice) caused an increase in aortic root lesional macrophage efferocytosis and a decrease in lesional necrotic area in comparison with the lesions of Phactr1 ${ }^{-/-} \rightarrow$ Phactr1 $^{-/-} \mathrm{Ldlr}^{-/-}$mice (Figure 7I), further supporting the idea that hematopoietic PHACTR1 helps stabilize plaques. Total lesion area was also lower in the Phactr1 $^{+/+} \rightarrow$ Phactr1 $^{\text {-/- }^{-}}$Ldlr $^{-/-}$cohort (Supplemental Figure 5A), while there were no significant differences between the 2 groups in body weight, plasma cholesterol, blood glucose, or blood monocytes (Supplemental Figure 5, B-E, respectively). In summary, these experimental atherosclerosis data, when considered together with the human lesional, HMDM, and mechanistic data, add support to the hypothesis that impaired macrophage efferocytosis conferred by PHACTR1 loss-of-expression polymorphisms contributes to a higher risk of atherosclerotic CAD.

\section{Discussion}

Defective efferocytosis and its consequences, including post-apoptotic cellular necrosis, cellular debris-mediated inflammation, and impaired resolution, are important drivers of clinically dangerous plaque features (7-10). While these studies have shown defective macrophage efferocytosis in advanced human coronary plaques, impaired efferocytosis has also been observed in macrophages derived from the circulating monocytes of CAD versus healthy patients in a manner that correlated with plaque severity (14). Among the mechanisms of defective efferocytosis in atherosclerosis are defects in one or more macrophage efferocytosis processes, i.e., AC binding, actin-mediated AC engulfment, and phagolysosomal AC degradation and processing (7-10). Concerning AC engulfment, most work has emphasized the roles of GTPases, particularly Rac1, and their cognate GTP-exchange factors, in orchestrating actin remodeling around the developing
AC phagosome (50-52). The work here shows that phosphorylation-activation of MLC, mediated by sequestration of PP1 $\alpha$ in the nucleus by PHACTR1, is also necessary for optimal AC engulfment by macrophages. To our knowledge, the role of MLC in efferocytosis has not previously been reported, but the role of myosin, particularly cortical actomyosin, in providing the force needed to form phagosomes has been widely studied (53). Indeed, the idea that MLC/myosin plays a similar role in efferocytosis is consistent with our data in Figure 3F showing that PHACTR1 silencing impairs phagosome formation around an AC.

Among the many CAD-risk loci that have been identified over the last decade, the PHACTR1 intronic rs9349379 risk allele $\mathrm{G}$ is notable for the reproducibility and strength of its association with CAD and its relatively high frequency $(17,19,23)$. The combination of these genetic data, the role of PHACTR1 in AC engulfment elucidated here, and the critical role of impaired efferocytosis in atherosclerosis progression suggested a mechanistic hypothesis linking the risk allele to CAD. The plausibility of this hypothesis is supported by our data linking the risk variant to lower expression of PHACTR1 in macrophages, particularly atherosclerotic lesional macrophages, which, to our knowledge, had not previously been shown in any cell type for PHACTR1 protein. The data demonstrating this point in HMDMs and human lesional macrophages may have a mechanistic basis in terms of regulation of PHACTR1 transcription, as previous data in human endothelial cells showed that the risk variant lowered PHACTR1 mRNA by compromising the binding of a transcription factor, myocyte enhancer factor-2 (MEF2), to a putative enhancer region in the intronic locus $(27,29)$. As MEF2 is also expressed in human macrophages (54), this mechanism of transcriptional repression may also be applicable to macrophages. Notably, a report showing lower expression of only a short form of PHACTR1 mRNA in HMDMs from rs9349379-GG subjects (39) is not consistent with our protein data and is discussed below.

Six transcripts have been reported to originate from the human PHACTR1 gene: long, intermediate-A+, intermediate-A-, intermediate- $\mathrm{B}+$, intermediate- $\mathrm{B}-$, and short $(39,55)$. The long transcript encodes a 580-amino acid protein with 4 actin-binding RPEL motifs and a PP1-binding domain, all of which are conserved in the protein translated from the long form of mouse Phactr1 mRNA (33, 36). Curiously, one of the aforementioned papers reported that the long transcript of PHACTR1 could not be detected in $\mathrm{CD} 14^{+}$monocyte-derived macrophages by PCR or immunoblotting, whereas it was the short transcript that was shown to be lower in monocyte-macrophages from subjects harboring rs9349379-GG by quantitative PCR (39); while one of the others reported that human macrophages express only the intermediate-B and short alleles by PCR, but did not look at the effect of rs9349379 genotype (55). In contrast, we were able to readily detect the long transcript by quantitative PCR and its encoded PHACTR1 protein isoform by immunoblot in HMDMs. We also showed that the long-form expression was lower in rs9349379-GG subjects, as was immunoreactive PHACTR1 protein colocalizing with macrophages in atherosclerotic lesions. Most importantly, the presence and functional importance of the long form of PHACTR1 in macrophages are implicated by our in vitro and in vivo molecular-genetic causation studies, as it is only this form that has both the NLS and PP1 $\alpha$-binding domains required for the efferocytic function of PHACTR1. 
While these combined data suggest that impaired efferocytosis might be a mechanism linking the risk variant to $\mathrm{CAD}$, we favor the idea that additional, complementary mechanisms are also involved, including those involving other consequences of decreased PHACTR1 in macrophages, decreased PHACTR1 in endothelial cells $(27,29,56)$, and possibly increased endothelin-1 in endothelial cells (28). Regarding other possible macrophage effects, Li et al. (32) reported recently that fat-fed $\mathrm{Apoe}^{-/-}$mice with an absence of hematopoietic PHACTR1 had increased atherosclerosis compared with Apoe $e^{-/}$mice with normal PHACTR1 expression. The authors ascribed this effect to the induction of an inflammatory signaling pathway in $\mathrm{Phactr}^{\mathrm{H}^{-/}}$macrophages. However, as efferocytosis was not examined in this report, future studies will be needed to determine whether this inflammatory pathway is a separate consequence of PHACTR1 deficiency or secondary to impaired efferocytosis, which is known to activate inflammatory pathways (57). Moreover, as neither PHACTR1 polymorphisms nor human atheroma was examined, the potential relevance of the mouse findings in this study to human atherosclerosis and the rs9349379 risk variant remains to be investigated. In considering the role(s) of PHACTR1 in endothelial cells, the consequences of lower PHACTR1 or higher endothelin-1 in these cells could be additive or synergistic with effects in macrophages, as these 2 cell types play complementary and interactive roles in atherosclerosis (2). Moreover, the endothelial hypothesis may also have relevance to non-atherosclerotic vascular diseases associated with rs9349379-G, including migraine headache, arterial dissection, fibromuscular dysplasia, hypertension, and coronary microvascular dysfunction $(28,58,59)$. Except for coronary microvascular dysfunction, the risk of these non-atherosclerotic vascular diseases is lower in carriers of the rs $9349379-G$ variant in contrast to the higher risk of CAD conferred by rs9349379-G, indicating that future work is needed to link the polymorphism to these diseases.

In summary, we present what we believe to be new roles for PHACTR1, MLC, and PP1 $\alpha$ in efferocytosis and show that the PHACTR1 intronic rs9349379 CAD-risk allele lowers the fully functional form of PHACTR1 protein and impairs efferocytosis in human atherosclerotic lesional macrophages and HMDMs. We also show direct causation and mechanistic evidence in hematopoietic Phactr1-targeted mice. When considered together with the possible atherogenic role of PHACTR1 deficiency in lesional endothelial cells, as well as a recent study showing lower PHACTR1 mRNA in human carotid atheroma versus normal carotid (31), our findings suggest that therapies that could enhance PHACTR1 expression, such as agonists of receptors that lead to PHACTR1 induction (37), may have promise as a new human genetics-based therapy for atherosclerosis and possibly other vascular diseases.

\section{Methods}

Cell lines. The Jurkat human T lymphocyte E6-1 cell line was obtained from ATCC and cultured in DMEM (GIBCO) supplemented with $10 \%$ (vol/vol) heat-inactivated FBS (HI-FBS; GIBCO), $10 \mathrm{U} / \mathrm{mL}$ penicillin, and $100 \mathrm{mg} / \mathrm{mL}$ streptomycin (Corning). Cells were cultured in a humidified $\mathrm{CO}_{2}$ incubator at $37^{\circ} \mathrm{C}$.

Bone marrow-derived macrophages. Mice were euthanized with isoflurane, and hind legs were removed. Femurs and tibiae were flushed with DMEM containing $4.5 \mathrm{~g} / \mathrm{L}$ glucose (GIBCO), 20\% L-929 fibro- blast-conditioned medium, 10\% HI-FBS, and 1\% penicillin/streptomycin using a 26-gauge needle. Cell suspensions were passed over a $40-\mu \mathrm{m}$ filter, centrifuged at $500 \mathrm{~g}$, resuspended in $50 \mathrm{~mL}$ of medium, and plated into five 100-mm dishes. Cells were incubated for 4 days, after which nonadherent cells and debris were aspirated and the medium was replaced with fresh medium. After 7-10 days of differentiation, with medium changed every 2-3 days, cells were harvested for use in various experiments.

Human monocyte-derived macrophages. Peripheral blood leukocytes were isolated from buffy coats of anonymous, deidentified healthy adult volunteers purchased from the New York Blood Center (NYBC), with informed consent obtained by the NYBC. In brief, the buffy coats were gently layered onto Histopaque solution (Sigma-Aldrich) and centrifuged at $1620 \mathrm{~g}$ for 30 minutes at room temperature. Leukocytes were removed from the middle layer, washed with RPMI 1640 (GIBCO) containing $10 \% \mathrm{FBS}$ and $1 \%$ penicillin/streptomycin, and then centrifuged at $1620 \mathrm{~g}$ for 5 minutes. This wash step was repeated once before resuspension of the pellet in RPMI 1640 medium and plating of cells into 12-well plates. After approximately 3-4 hours, when more than $50 \%$ of the cells were adherent, the medium was changed to RPMI 1640 medium supplemented with $10 \mathrm{ng} / \mathrm{mL}$ GM-CSF (PeproTech). The medium was replaced with fresh GM-CSF-containing medium every 2 days. The macrophages were used for experiments between days 7 and 14, at which time the cells were more than $80 \%$ confluent.

Single-cell RNA-Seq data analysis. Expression matrices of human atherosclerotic carotid artery single-cell RNA-Seq (scRNA-Seq) from 3 subjects were downloaded from Gene Expression Omnibus (GSE155512). The deidentified carotid artery specimens were obtained from patients undergoing carotid endarterectomy for highgrade stenosis of the internal carotid artery at Columbia University Irving Medical Center. The use of these specimens, which were removed for clinical indications only and would otherwise have been discarded, was approved by the Columbia University IRB, and procedures were conducted in accordance with an approved IRB protocol. Informed consent was obtained from each patient. Data filtering, integration, and clustering analysis were performed in Seurat 3.1.1 (60). The following gene-level and cell-level filtering was applied to each sample: (a) included genes expressed in $\geq 10$ cells; (b) included cells that had 200 to 4000 genes expressed and had $\leq 20,000$ unique molecular identifiers (UMIs); (c) included cells that had $\leq 10 \%$ reads mapped to mitochondrial genes. Filtered data sets were integrated using SCTransform workflow (https://cran.r-project.org/web/packages/Seurat/index.html). Briefly, 1000 variable genes were selected from each data set, and the top 1500 variable genes across 3 data sets were used as integration features. Integration anchors were identified using the first 20 dimensions from canonical correlation analysis. Uniform manifold approximation and projection (UMAP) visualization was generated using the first 20 principal components (PCs). A shared nearest neighbor (SNN) graph was constructed using 30 nearest neighbors and 20 dimensions of PCs. Louvain clustering was then performed with a resolution parameter of 0.6. Differential expression analysis was performed on cells from each cluster compared with all other cells using the Model-based Analysis of Single Cell Transcriptomics (MAST) test implemented in the R package Seurat, where each gene was required to be present in at least $25 \%$ of the cells in either group. A gene was considered differentially expressed if it had fold change $\geq 1.5$ and Bonferroni corrected $P$ value less than 0.05. 
Animal husbandry. Mice were socially housed in standard cages at $22^{\circ} \mathrm{C}$ on a 12 -hour light/12-hour dark cycle in a barrier facility with ad libitum access to water and food. Sperm of C57BL/6NCrl-Phactr1 ${ }^{--}$was purchased from UC Davis KOMP Repository (MMRRC:043404-UCD), and in vitro fertilization was conducted by Charles River Laboratories using C57BL/6J eggs. Phactr1 ${ }^{+/}$pups were sent to Columbia University's animal facility, where the mice were backcrossed for more than 10 generations with C57BL/6J mice (The Jackson Laboratory, stock 000664). Phactr1 $^{+/-}$mice on the C57BL/6J background were cross-bred to obtain wild-type (Phactr1 $\left.{ }^{+/+}\right)$, Phactr1 $^{+/}$, and $\mathrm{Phactr}^{1^{-/}}$mice for bone marrow transplantation into $\mathrm{Ldlr} \mathrm{r}^{-/}$mice (The Jackson Laboratory, stock 000227). $\mathrm{Ldlr}^{-/}$recipient mice were randomly assigned to experimental groups by investigators. Phactr1 ${ }^{-1}$ mice were crossed with $\mathrm{Ldlr}^{-/}$mice (The Jackson Laboratory, stock 000227) to obtain $\mathrm{Ldll}^{-/}$Phactr1 ${ }^{-/-}$as recipient mice for bone marrow transplantation from wild-type $\left(\right.$ Phactr $\left.^{+/+}\right)$and Phactr1 ${ }^{-/-}$mice. Investigators were blinded for the atherosclerosis studies but were not blinded for the dexamethasone-induced thymus injury experiments.

Induction of apoptosis and fluorescent labeling of Jurkat cells. Jurkat cells were irradiated under a 254-nm UV lamp for 15 minutes, followed by incubation under normal cell culture conditions for 2-3 hours. The cells were then rinsed once with serum-free DMEM, resuspended at a concentration of $2 \times 10^{7}$ cells $/ \mathrm{mL}$ in Diluent C (Sigma-Aldrich), and stained with PKH26 dye (Sigma-Aldrich) according to the manufacturer's protocol. The cells were then rinsed twice with DMEM containing 10\% HI-FBS and used for experiments. This method routinely typically yields $>85 \%$ annexin $\mathrm{V}^{+}$ACs.

LPS and IFN- $\gamma$ stimulation of macrophages. BMDMs from WT, Phactr1 ${ }^{+/}$, and Phactr1 ${ }^{-/}$mice or siPHACTR1-treated HMDMs were plated at $0.2 \times 10^{6}$ cells per well on 24 -well plates and allowed to adhere overnight. The following day, cells were exposed to medium containing $1 \mathrm{ng} / \mathrm{mL}$ LPS (Sigma-Aldrich) and $20 \mathrm{ng} / \mathrm{mL}$ IFN- $\gamma$ (PeproTech) or vehicle control. After incubating for 24 hours, the cells were either incubated with ACs to assay efferocytosis or subjected to immunoblot analysis.

In vitro efferocytosis assay. BMDMs or HMDMs were plated in 24 -well dishes at a density of $0.18 \times 10^{6}$ cells per well. $\mathrm{PKH} 26$-labeled ACs were added to the wells at a 5:1 AC/macrophage ratio. After 45 minutes of incubation, the macrophage monolayers were rinsed 3 times with PBS and then fixed with $4 \%$ formaldehyde for 20 minutes, rinsed 3 times with PBS, and imaged on a Leica epifluorescence microscope (DMI6000B).

DiI-oxLDL uptake assay. BMDMs were plated in 24-well dishes at a density of $0.2 \times 10^{6}$ cells per well and allowed to adhere overnight. The following day, the cells were incubated with $10 \mu \mathrm{g} / \mathrm{mL}$ DiI-labeled oxidized LDL (DiI-oxLDL; Invitrogen). After 4 hours, cells were rinsed with PBS and fixed with $4 \%$ formaldehyde for 15 minutes. Cells were rinsed again, counterstained with Hoechst $(1 \mu \mathrm{g} / \mathrm{mL}$; Cell Signaling Technology) for 15 minutes, and imaged on a Leica epifluorescence microscope (DMI6000B).

Immunoblotting and immunofluorescence microscopy of macrophages. Macrophages were lysed in $2 \times$ Laemmli lysis buffer (Bio-Rad) containing $50 \mathrm{mM}$ DTT. Cell lysates were boiled for 5 minutes and then separated on 4\%-20\% SDS-PAGE gradient gels (Invitrogen) at $120 \mathrm{~V}$ for $1.5-2$ hours, and electrotransferred to $0.45-\mathrm{mm}$ nitrocellulose membranes at $250 \mathrm{~A}$ for $1-2$ hours. The membranes were incubated overnight at $4^{\circ} \mathrm{C}$ with 1:1000 anti-PHACTR1 (Novus, NBP1-84232, for HMDMs [Figure 1]; Abcam, ab229120, for BMDMs and HMDMs [Figure 2]), anti-GAPDH (Cell Signaling Technology, 8884S), antiMLC (Abcam, ab79935), anti-PP1 (Abcam, ab150782), and anti-MYC (Cell Signaling Technology, 2272S) diluted in PBS containing 1\% BSA, and detected using HRP-conjugated secondary antibodies (Pierce). Densitometry was performed using ImageJ software (NIH). For immunofluorescence microscopy, macrophages were incubated with ACs at a $5: 1 \mathrm{AC} /$ macrophage ratio for 20 minutes and then fixed in $4 \%$ formaldehyde, permeabilized with $0.1 \%$ Triton $\mathrm{X}-100$, blocked for 1 hour with $1 \%$ denatured BSA, and incubated with 1:100 anti-MLC2 (Abcam, ab79935), anti-phospho-MLC2 (Cell Signaling Technology, 3671), anti-PHACTR1 (Abcam, ab229120), and anti-PP1 $\alpha$ (Abcam, ab150782) antibodies for at least 18 hours. Cells were rinsed with TBS with $0.1 \%$ Tween-20 (TBST) and incubated for 2 hours with 1:200 Alexa Fluor 488- and Alexa Fluor 647-conjugated secondary antibodies (BioLegend). Unbound secondary antibodies were removed by rinsing 3 times with TBST, and then the cells were incubated with Hoechst stain (1 $\mu \mathrm{g} / \mathrm{mL}$; Cell Signaling Technology) for 15 minutes followed by another rinsing with TBST. Then cells were visualized and image-captured using a Leica epifluorescence microscope (DMI6000B).

Confocal microscopy imaging. Scrambled RNA or Phactr1 siRNA was electroporated into BMDMs with $1.5 \mu \mathrm{g}$ of LifeAct-RFP (mTagRFP-T-Lifeact-7; Addgene plasmid 54586) using the Neon Invitrogen Electroporation kit (Invitrogen). BMDMs were subjected to 1650 $\mathrm{V}$ at 10-millisecond intervals for 2 pulses within the electroporation chamber, then plated onto 8-well glass-bottom coverslips and allowed to adhere for 18-24 hours. ACs were then added at a 3:1 ratio of ACs to macrophages for an additional 45 minutes, after which the macrophages were rinsed with PBS and then fixed with $4 \%$ paraformaldehyde for 15 minutes at room temperature. Imaging was conducted on a Zeiss LSM 880 NLO confocal microscope with an Airyscan module (Carl Zeiss Microscopy) equipped with a $\times 63$ oil 1.4 numerical aperture (NA) objective lens and collected through a 32-channel GaAsP detector as 0.2 Airy units per channel. $Z$-stack images were acquired at an average of 30 slices, with a $0.17-\mu \mathrm{m}$ distance between each slice.

siRNA-mediated gene silencing. Scrambled siRNA control and gene-targeting siRNAs (Integrated DNA Technologies) were transfected into macrophages using INTERFERin siRNA transfection reagent (Polyplus-transfection) in 24-well plates following the manufacturer's instructions. To downregulate all isoforms of PHACTR1, a pool of siRNAs was used targeting exons 3, 14, and 15 of the human PHACTR1 gene, or exons 11, 12, and 13 of the murine Phactr1 gene. Cells were incubated for 6 hours with $1.5 \mathrm{~mL}$ of Opti-MEM (GIBCO) containing INTERFERin siRNA transfection reagent, $0.18 \times 10^{6}$ cells, and $10 \mathrm{nM}$ siRNA. Experiments and protein analyses were conducted 3 days later.

Quantitative PCR. RNA was isolated from macrophages using the RNeasy Kit (QIAGEN), and cDNA was synthesized from RNA, which ranged from 500 ng to $1 \mu \mathrm{g}$ per reaction, using a cDNA synthesis kit (Applied Biosystems). Real-time PCR was conducted using a 7500 Real-Time PCR System and SYBR Green reagents (Applied Biosystems). Human primer sets used were as follows: PHACTR1 forward, GCGGCATCCTCGGAGGATG; PHACTR1 reverse, TCACTGGCAGACAAGGCAAT; HPRT forward, CCTGGCGTCGTGATTAGTGAT; and HPRT1 reverse, AGACGTTCAGTCCTGTCCATAA.

Immunoprecipitation of PP1 $\alpha$ from macrophage nuclei. BMDMs were seeded to $100-\mathrm{mm}$ culture dishes and were incubated in the presence or absence of ACs for 20 minutes at $37^{\circ} \mathrm{C}$. Cells were 
removed from the dishes, added to $0.5 \mathrm{~mL}$ of ice-cold cytoplasmic extraction reagent of the NE-PER Nuclear and Cytoplasmic Extraction Kit (Thermo Fisher Scientific), and incubated on ice for 10 minutes. Then cell lysates were centrifuged at $14,000 \mathrm{~g}$ for 5 minutes at $4^{\circ} \mathrm{C}$, and the pellets were resuspended with $0.25 \mathrm{~mL}$ of the nuclear extraction reagent of the kit and incubated for 40 minutes, followed by centrifugation at $14,000 \mathrm{~g}$ for 10 minutes at $4^{\circ} \mathrm{C}$. The resulting supernatant fraction was preabsorbed using $20 \mu \mathrm{L}$ Protein $\mathrm{G}$ magnetic beads (Cell Signaling Technology) and then incubated overnight with anti-PP1 $\alpha$ (Thermo Fisher Scientific, 43-8100) antibody while gently rotating at $4^{\circ} \mathrm{C}$. Rabbit IgG was used as a negative control. Twenty microliters of Protein G magnetic beads were added to the solution, and after incubation for 20 minutes at room temperature, the beads were collected using a magnetic rack. The beads were then rinsed 5 times with $500 \mu \mathrm{L}$ cell lysis buffer (Cell Signaling Technology), suspended in $40 \mu \mathrm{L}$ of $4 \times$ Laemmli Sample Buffer (Bio-Rad), and subjected to immunoblot analysis.

Genotyping of rs9349379. Genomic DNA was isolated from HMDMs or carotid artery tissue using a genomic DNA extraction kit from Invitrogen per the manufacturer's instructions. DNA was genotyped using TaqMan SNPs Genotyping Assay (Thermo Fisher Scientific) for rs9349379 per the manufacturer's instructions.

Plasmids and electroporation. cDNAs for the mouse Phactr1 and $M l c 2$ genes were synthesized and cloned into the pcDNA3.1-c-Myc vector using EcoRI and XhoI restriction enzymes (GenScript) (36). Phactr1 mutants were derived from the pcDNA3.1-c-Myc-Phac$\operatorname{tr} 1$ vector by deletion of $\mathrm{B} 1$ to delete the NLS site C to delete the PP1 $\alpha$-binding site (GenScript). The $M l c 2$ mutant was derived from the pcDNA3.1-c-Myc-MLC vector by mutation of S18 and S19 sites to aspartic acid. The plasmids $(2 \mu \mathrm{g})$ were electroporated into $2 \times 10^{6}$ Phactr1 $1^{-/}$BMDMs (for Phactr1 mutants) or $2 \times 10^{6}$ Phactr1 $^{+/+}$and Phactr1 $1^{-/-}$BMDMs (for Mlc2 mutant) using the Neon Transfection System from Invitrogen. Pellets of BMDMs and DNA were resuspended in $10 \mu \mathrm{L}$ Buffer R (Invitrogen) and subjected to electroporation at $1550 \mathrm{~V}$ at 10 -millisecond intervals for 2 pulses. Macrophages were then plated into 24-well culture dishes, and experiments and protein analyses were conducted 3 days later.

Blood and plasma analyses. Fasting blood glucose was measured using a glucose meter (Lifescan, OneTouch Ultra) in mice that were fasted for 5 hours, with free access to water. Standard kits were used to measure plasma triglycerides (Wako, Triglyceride M kit) and cholesterol (Wako, Cholesterol E kit) per the manufacturer's instructions. Complete blood cell count, including leukocyte differential, was obtained using a FORCYTE Hematology Analyzer (Oxford Science).

Mouse atherosclerosis study. Eight-week-old male $\mathrm{Ldlr}^{--}$and $\mathrm{Ldlr}^{-1}$ Phactr1 ${ }^{-/-}$mice were irradiated with $10 \mathrm{~Gy}$ from a cesium-137 $\gamma$-emitting irradiator (Gamma Cell 40, MSD Nordion). Four hours after irradiation, the mice were injected via tail vein with $5 \times 10^{6}$ bone marrow cells from 16- to 20-week-old male Phactr1 ${ }^{+/+}$and Phactr1 ${ }^{-/}$mice. The bone marrow cells were prepared as follows: Femurs from donor mice were isolated and flushed with DMEM containing BMDM cell medium. The cells were filtered through a $40-\mu \mathrm{m}$ nylon cell and centrifuged at $600 \mathrm{~g}$ for 10 minutes at room temperature. Bone marrow cells were rinsed twice, and the pellets were diluted in DMEM. Mice were given water containing neomycin $(0.1 \mathrm{mg} / \mathrm{mL}$; Sigma-Aldrich) for 2 weeks after bone marrow transplantation (BMT). Four weeks after BMT, mice were placed on a Western diet (WD; Envigo) for 8 weeks (for the Phactr1 ${ }^{+/+}$vs. Phactr1 $1^{-/-}$BMT study and the Phactr1 $1^{+/+}$ vs. Phactr $1^{-/-}$reverse BMT study in Ldlr $^{-/}$Phactr1 $1^{-/}$mice) or 16 weeks (for the Phactr $1^{+/+}$vs. Phactr1 ${ }^{+/}$BMT study). At the time of harvest, mice were euthanized using isoflurane. Blood was removed by left ventricular puncture, and the vasculature was then perfused with cold PBS. Aortic roots were fixed in paraformaldehyde and paraffin-embedded. Serial $6-\mu \mathrm{m}$ sections were obtained for analysis. For morphometric analysis, 6 paraffin sections $60 \mu \mathrm{m}$ apart were stained with Harris's H\&E. Total lesion and necrotic core areas were defined as previously described (61). Collagen staining was performed using Picrosirius red (Polysciences) per the manufacturer's instructions. Collagen cap thickness was quantified from 3 distinct regions of the plaque as previously described (61). Sections were deparaffinized and subjected to antigen retrieval using $10 \mathrm{mM}$ Tris, 0.5 M EDTA, $0.05 \%$ Tween-20. For the identification of TUNEL ${ }^{+}$cells, the TUNEL staining kit from Roche was used, using a staining period of 1 hour at $37^{\circ} \mathrm{C}$. For immunostaining, sections were blocked for 1 hour using serumfree protein blocking buffer (DAKO, catalog X0909) and then incubated overnight at $4^{\circ} \mathrm{C}$ with the following primary antibodies: Mac2 (Cedarlane, CL8942LE; 1:1000), phospho-MLC2 (Cell Signaling Technology, 3671; 1:100), and total MLC2 (Abcam, ab79935; 1:100). Two slides, each with 2 sections, were assessed for each mouse. Parallel slides were used for staining with isotype controls. After rinsing in PBS, slides were incubated with secondary antibodies for 2 hours at room temperature and were counterstained with DAPI. In situ efferocytosis was quantified as described below. Mean fluorescence intensity (MFI) of phospho-MLC was quantified in $\mathrm{Mac2}^{+}$cells that were associated with TUNEL ${ }^{+}$cells and expressed relative to total MLC MFI. All images were captured using a Zeiss fluorescence microscope and analyzed using Image J.

Human plaque analysis. Deidentified carotid artery specimens were obtained from patients undergoing carotid endarterectomy for high-grade stenosis of the internal carotid artery at the Division of Vascular Surgery, University Medical Center, Johannes Gutenberg University (Mainz, Germany). After retrieval in the operating room, the excised plaques were rinsed in physiologic saline, immediately snap-frozen in liquid nitrogen, and then stored at $-80^{\circ} \mathrm{C}$. After thawing, samples were fixed in $4 \%$ formalin overnight, decalcified in EDTA for 4 days, and then embedded in paraffin blocks. Serial sections were obtained at $5-\mu \mathrm{m}$ intervals. Before immunofluorescence staining, all sections were deparaffinized in xylene and then rehydrated in a graded series of ethanol concentrations. Sections were then boiled for $20 \mathrm{~min}-$ utes in an antigen retrieval buffer (10 mM Tris, 0.5 M EDTA, 0.05\% Tween-20). After rinsing in PBS, sections were stained for TUNEL ${ }^{+}$ cells as above. After incubation in serum-free protein blocking buffer (DAKO, catalog X0909) for 1 hour, sections were incubated overnight at $4^{\circ} \mathrm{C}$ with primary antibody directed toward CD68 (DAKO, catalog M0814; 1:100) for the in situ efferocytosis assay described below. For PHACTR1 staining, sections were incubated with anti-PHACTR1 antibody (Abcam, ab229120; 1:100) overnight at $4^{\circ} \mathrm{C}$. The sections were then rinsed in PBS and incubated with secondary antibodies for 2 hours at room temperature. Parallel slides were used for staining with isotype controls. Slides were mounted with DAPI-containing mounting solution, and images were obtained using a Leica microscope and analyzed using Fiji/ImageJ software (NIH). Two slides, each with 2 sections, were assessed for each patient. To evaluate whether the risk allele had a primary effect on decreasing PHACTR1 expression and 
efferocytosis by lesional macrophages, the lesions were selected to be similarly advanced in terms of plaque necrosis, i.e., to make sure that any effects seen were not simply secondary to an effect of the risk allele on causing more advanced plaques.

In situ efferocytosis. Mouse aortic roots, human carotid arteries, or mouse thymus sections were assessed for efferocytosis by counting of TUNEL ${ }^{+}$nuclei that were associated with $\mathrm{Mac}^{+}$macrophages ("associated"), indicative of efferocytosis, or not associated with macrophages ("free") (61). Macrophage-associated TUNEL ${ }^{+}$cells were defined as $\mathrm{TUNEL}^{+}$nuclei surrounded by or in contact with $\mathrm{Mac}^{+}$macrophages. Free ACs exhibited nuclear condensation and loss of antibody Mac2 reactivity and were not in contact with neighboring macrophages.

In vivo dexamethasone thymus assay. Eight-week-old male Phactr1 ${ }^{+/+}$, Phactr1 $^{+/-}$, and Phact1r ${ }^{-/}$mice were injected i.p. with $250 \mathrm{~mL}$ PBS containing $250 \mathrm{mg}$ dexamethasone (Sigma-Aldrich) or PBS control. Eighteen hours after injection, the mice were euthanized, and thymi were harvested and weighed. One lobe of the thymus from each mouse was mechanically disaggregated, and cells were enumerated. Flow cytometry was then conducted to determine the number of annexin $\mathrm{V}^{+}$cells. The other thymus lobe was formalin-fixed, paraffin-embedded, and sectioned, followed by staining of the sections (5 mm) with TUNEL reagents (Roche) and antibodies against Mac2 (Cedarlane, CL8942LE; 1:1000), phospho-MLC2 (Cell Signaling Technology, 3671; 1:100), and total MLC2 (Abcam, ab79935; 1:100). For phospho-MLC2 quantification, thymus sections were stained with TUNEL reagent, anti-phospho-MLC2, and anti-total MLC2. Mac2 ${ }^{+}$cells either engulfing or contacting TUNEL ${ }^{+}$ACs were chosen, in which MFI of phospho-MLC2 was measured and normalized to $\mathrm{Mac}^{+}$cells total MLC MFI.

Flow cytometric analysis of ACs. Cells were washed twice with cold FACS buffer (PBS containing 2\% FBS and $1 \mathrm{mM}$ EDTA), resuspended in annexin V-binding buffer (BioLegend) at a concentration of $1 \times 10^{6}$ cells/mL, and incubated with FITC-conjugated annexin V (BioLegend) for 15 minutes at room temperature. Samples were then analyzed on a BD FACSCanto II flow cytometer. Data analysis was carried out using FlowJo software.

Quantification and statistical analysis. Data were tested for normality using the Kolmogorov-Smirnov test, and statistical significance was determined using GraphPad Prism software. Data that passed the normality test were analyzed using 2-tailed Student's $t$ test for 2 groups with 1 variable tested and equal variances, 1-way ANOVA with Dunnett's multiple-comparison test for multiple groups with only 1 variable tested, or 2-way ANOVA with Bonferroni post-tests for more than 2 groups with multiple variables tested. Data that were not normally distributed but contained equal variances were analyzed using the nonparametric Mann-Whitney $U$ test with post hoc analysis. Data are shown as mean values \pm SEM. Differences were considered statistically significant at $P$ less than 0.05 .

Study approval. The Columbia University's Institutional Review Board and Health Insurance Portability and Accountability Act guidelines were followed for isolating peripheral human blood leukocytes. Mouse protocols were approved by Columbia University's IACUC. All mice were cared for according to the NIH guidelines for the care and use of laboratory animals, and all were in good general health based on appearance and activity. The use of deidentified carotid artery specimens, which were removed for clinical indications only and would otherwise have been discarded, conformed with the declaration of Helsinki and were approved for use by the Johannes Gutenberg University ethics review board. Informed written consent was obtained from each patient. The Columbia University IRB provided ethical approval for these studies, and procedures were conducted in accordance with an approved IRB protocol.

\section{Author contributions}

CK, DS, HZ, MPR, and IT conceived and designed the research. $\mathrm{BD}$ donated patient samples and was instrumental in the interpretation of the human data. CK, MS, BG, CX, XW, and GK conducted the experiments. CK, MS, BG, CX, ZZ, GF, GK, HZ, MPR, and IT analyzed the data. CK, MS, and IT wrote the paper, with comments provided by all other authors.

\section{Acknowledgments}

This work was supported by American Heart Association postdoctoral fellowship grant 20POST35210962 (to CK), the Niels Stensen Fellowship (to MS), NIH T32 training grant HL007343-28 (to BG), an American Society of Hematology Scholar Award and an American Heart Association Career Development Award (to ZZ), NIH grant HL141127 (to GF), NIH grants R00-HL130574 and R01-HL151611 (to HZ), NIH grants HL113147 and HL150359 (to MPR), and NIH R35 grant HL145228 (to IT). These studies used the resources of the Columbia Cancer Center flow core facilities, funded in part through NIH grants P30-CA013696, P30-CA013696, and S10-RR027050. The graphical abstract was created using BioRender.

Address correspondence to: Ira Tabas, Department of Medicine, Columbia University Irving Medical Center, 630 W. 168th Street, New York, New York 10032, USA. Phone: 212.305.9430; E-mail: iat1@columbia.edu.
1. Virani SS, et al. Heart disease and stroke statistics-2020 update: a report from the American Heart Association. Circulation. 2020;141(9):e139-e596.

2. Lusis AJ. Atherosclerosis. Nature. 2000;407(6801):233-241.

3. Tabas I, et al. Subendothelial lipoprotein retention as the initiating process in atherosclerosis: update and therapeutic implications. Circulation. 2007;116(16):1832-1844.

4. Glass CK, Witztum JL. Atherosclerosis. the road ahead. Cell. 2001;104(4):503-516.

5. Kasikara C, et al. The role of non-resolving inflammation in atherosclerosis. J Clin Invest. 2018;128(7):2713-2723.

6. Libby P, Hansson GK. Biology of disease: involvement of the immune system in human atherogenesis-current knowledge and unanswered questions. Lab Invest. 1991;64(1):5-15.

7. Yurdagul A Jr., et al. Mechanisms and consequences of defective efferocytosis in atherosclerosis. Front Cardiovasc Med. 2017;4:86.

8. Linton MF, et al. Macrophage apoptosis and efferocytosis in the pathogenesis of atherosclerosis. Circ J. 2016;80(11):2259-2268.

9. Schrijvers DM, et al. Phagocytosis of apop- totic cells by macrophages is impaired in atherosclerosis. Arterioscler Thromb Vasc Biol. 2005;25(6):1256-1261.

10. Kojima Y, et al. The role of efferocytosis in atherosclerosis. Circulation. 2017;135(5):476-489.

11. Kolodgie FD, et al. Pathologic assessment of the vulnerable human coronary plaque. Heart. 2004;90(12):1385-1391.

12. Libby P. Mechanisms of acute coronary syndromes and their implications for therapy. $N$ Engl JMed. 2013;368(21):2004-2013.

13. Fredman G, et al. An imbalance between specialized pro-resolving lipid mediators and 
pro-inflammatory leukotrienes promotes instability of atherosclerotic plaques. Nat Commun. 2016;7:12859.

14. Eligini S, et al. Biological profile of monocyte-derived macrophages in coronary heart disease patients: implications for plaque morphology. $S c i$ Rep. 2019;9(1):8680.

15. Musunuru K, Kathiresan S. Genetics of common, complex coronary artery disease. Cell. 2019;177(1):132-145.

16. McPherson R, Tybjaerg-Hansen A. Genetics of coronary artery disease. Circ Res. 2016;118(4):564-578.

17. Deloukas P, et al. Large-scale association analysis identifies new risk loci for coronary artery disease. Nat Genet. 2013;45(1):25-33.

18. Howson JMM, et al. Fifteen new risk loci for coronary artery disease highlight arterial-wall-specific mechanisms. Nat Genet. 2017;49(7):1113-1119.

19. Nikpay M, et al. A comprehensive 1,000 Genomes-based genome-wide association meta-analysis of coronary artery disease. Nat Genet. 2015;47(10):1121-1130.

20. Samani NJ, et al. Genomewide association analysis of coronary artery disease. N Engl J Med. 2007;357(5):443-453.

21. van der Harst P, Verweij N. Identification of 64 novel genetic loci provides an expanded view on the genetic architecture of coronary artery disease. Circ Res. 2018;122(3):433-443.

22. Kojima Y, et al. Cyclin-dependent kinase inhibitor $2 \mathrm{~B}$ regulates efferocytosis and atherosclerosis. J Clin Invest. 2014;124(3):1083-1097.

23. Kathiresan S, et al. Genome-wide association of early-onset myocardial infarction with single nucleotide polymorphisms and copy number variants. Nat Genet. 2009;41(3):334-341.

24. Hager J, et al. Genome-wide association study in a Lebanese cohort confirms PHACTR1 as a major determinant of coronary artery stenosis. PLOS One. 2012;7(6):e38663.

25. Nelson CP, et al. Association analyses based on false discovery rate implicate new loci for coronary artery disease. Nat Genet. 2017;49(9):1385-1391.

26. Lu X, et al. Genome-wide association study in Han Chinese identifies four new susceptibility loci for coronary artery disease. Nat Genet. 2012;44(8):890-894.

27. Beaudoin $\mathrm{M}$, et al. Myocardial infarction-associated SNP at 6p24 interferes with MEF2 binding and associates with PHACTR1 expression levels in human coronary arteries. Arterioscler Thromb Vasc Biol. 2015;35(6):1472-1479.

28. Gupta RM, et al. A genetic variant associated with five vascular diseases is a distal regulator of endothelin-1 gene expression. Cell. 2017;170(3):522-533.

29. Wang X, Musunuru K. Confirmation of causal rs9349379- PHACTR1 expression quantitative trait locus in human-induced pluripotent stem cell endothelial cells. Circ Genom Precis Med. 2018;11(10):e002327.

30. GTEx Consortium. The Genotype-Tissue Expression (GTEx) pilot analysis: multitissue gene regulation in humans. Science. 2015;348(6235):648-660.

31. Green IE, et al. Differential expression of PHACTR1 in atheromatous versus normal carotid artery tissue. J Clin Neurosci. 2020;74:265-267.

32. Li T, et al. Genetic deficiency of Phactr1 promotes atherosclerosis development via facilitating M1 macrophage polarization and foam cell formation. Clin Sci (Lond). 2020;134(17):2353-2368.

33. Allen PB, et al. Phactrs 1-4: a family of protein phosphatase 1 and actin regulatory proteins. Proc Natl Acad Sci U S A. 2004;101(18):7187-7192.

34. Sagara J, et al. Scapinin, a putative protein phosphatase-1 regulatory subunit associated with the nuclear nonchromatin structure. J Biol Chem. 2003;278(46):45611-45619.

35. Mouilleron S, et al. Structures of the Phactr1 RPEL domain and RPEL motif complexes with G-actin reveal the molecular basis for actin binding cooperativity. Structure. 2012;20(11):1960-1970.

36. Wiezlak M, et al. G-actin regulates the shuttling and PP1 binding of the RPEL protein Phactr1 to control actomyosin assembly. JCell Sci. 2012;125(pt 23):5860-5872.

37. Allain B, et al. Neuropilin-1 regulates a new VEGF-induced gene, Phactr-1, which controls tubulogenesis and modulates lamellipodial dynamics in human endothelial cells. Cell Signal. 2012;24(1):214-223.

38. Favot L, et al. Overexpression of a family of RPEL proteins modifies cell shape. FEBS Lett. 2005;579(1):100-104.

39. Reschen ME, et al. Genetic and environmental risk factors for atherosclerosis regulate transcription of phosphatase and actin regulating gene PHACTR1. Atherosclerosis. 2016;250:95-105.

40. Poon IK, et al. Apoptotic cell clearance: basic biology and therapeutic potential. Nat Rev Immunol. 2014;14(3):166-180

41. Müller A, et al. Imaging atherosclerotic plaque inflammation via folate receptor targeting using a novel $18 \mathrm{~F}$-folate radiotracer. Mol Imaging. 2014;13:1-11.

42. Lin JD, et al. Single-cell analysis of fate-mapped macrophages reveals heterogeneity, including stem-like properties, during atherosclerosis progression and regression. JCI Insight. 2019;4(4):124574.

43. Cochain C, et al. Single-cell RNA-seq reveals the transcriptional landscape and heterogeneity of aortic macrophages in murine atherosclerosis. Circ Res. 2018;122(12):1661-1674.

44. Thorp E, et al. Mertk receptor mutation reduces efferocytosis efficiency and promotes apoptotic cell accumulation and plaque necrosis in ath- erosclerotic lesions of apoe-/- mice. Arterioscler Thromb Vasc Biol. 2008;28(8):1421-1428.

45. Sun B, et al. Distinct mechanisms for OxLDL uptake and cellular trafficking by class B scavenger receptors CD36 and SR-BI. J Lipid Res. 2007;48(12):2560-2570.

46. Kaneko-Kawano T, et al. Dynamic regulation of myosin light chain phosphorylation by Rhokinase. PLoS One. 2012;7(6):e39269.

47. Matsumura F, Hartshorne DJ. Myosin phosphatase target subunit: many roles in cell function. Biochem Biophys Res Commun. 2008;369(1):149-156.

48. Ito M, et al. Myosin phosphatase: structure, regulation and function. Mol Cell Biochem. 2004;259(1-2):197-209.

49. Park D, et al. Continued clearance of apoptotic cells critically depends on the phagocyte Ucp2 protein. Nature. 2011;477(7363):220-224.

50. Elliott MR, Ravichandran KS. The dynamics of apoptotic cell clearance. Dev Cell. 2016;38(2):147-160.

51. Proto JD, et al. Regulatory T cells promote macrophage efferocytosis during inflammation resolution. Immunity. 2018;49(4):666-677.

52. Yurdagul A Jr., et al. Macrophage metabolism of apoptotic cell-derived arginine promotes continual efferocytosis and resolution of injury. Cell Metab. 2020;31(3):518-533.

53. Barger SR, et al. Squeezing in a meal: myosin functions in phagocytosis. Trends Cell Biol. 2020;30(2):157-167.

54. Aude-Garcia C, et al. Dual roles for MEF2A and MEF2D during human macrophage terminal differentiation and c-Jun expression. Biochem J. 2010;430(2):237-244.

55. Codina-Fauteux VA, et al. PHACTR1 splicing isoforms and eQTLs in atherosclerosis-relevant human cells. BMC Med Genet. 2018;19(1):97.

56. Jarray R, et al. Disruption of phactr-1 pathway triggers pro-inflammatory and pro-atherogenic factors: new insights in atherosclerosis development. Biochimie. 2015;118:151-161.

57. Lemke G, Rothlin CV. Immunobiology of the TAM receptors. Nat Rev Immunol. 2008;8(5):327-336.

58. Ford TJ, et al. Genetic dysregulation of endothelin- 1 is implicated in coronary microvascular dysfunction. Eur Heart J. 2020;41(34):3239-3252.

59. Saw J, et al. Chromosome 1q21.2 and additional loci influence risk of spontaneous coronary artery dissection and myocardial infarction. Nat Commun. 2020;11(1):4432.

60. Butler A, et al. Integrating single-cell transcriptomic data across different conditions, technologies, and species. Nat Biotechnol. 2018;36(5):411-420.

61. Fredman G, et al. Targeted nanoparticles containing the pro-resolving peptide Ac2-26 protect against advanced atheosclerosis in hypercholesterolemic mice. Sci Transl Med. 2015;7(275):275ra20. 